\title{
About the Properties of a Modified Generalized Beverton-Holt Equation in Ecology Models
}

\author{
M. De La Sen \\ Department of Electricity and Electronics, Institute for Research and Development of Processes, \\ Faculty of Science and Technology, University of the Basque Country, Campus of Leioa, \\ 544 Bilbao, 48940 Leioa, Spain \\ Correspondence should be addressed to M. De La Sen, manuel.delasen@ehu.es \\ Received 5 February 2008; Revised 10 April 2008; Accepted 22 April 2008 \\ Recommended by Antonia Vecchio
}

This paper is devoted to the study of a generalized modified version of the well-known BevertonHolt equation in ecology. The proposed model describes the population evolution of some species in a certain habitat driven by six parametrical sequences, namely, the intrinsic growth rate (associated with the reproduction capability), the degree of sympathy of the species with the habitat (described by a so-called environment carrying capacity), a penalty term to deal with overpopulation levels, the harvesting (fishing or hunting) regulatory quota, or related to use of pesticides when fighting damaging plagues, and the independent consumption which basically quantifies predation. The independent consumption is considered as a part of a more general additive disturbance which also potentially includes another extra additive disturbance term which might be attributed to net migration from or to the habitat or modeling measuring errors. Both potential contributions are included for generalization purposes in the proposed modified generalized Beverton-Holt equation. The properties of stability and boundedness of the solution sequences, equilibrium points of the stationary model, and the existence of oscillatory solution sequences are investigated. A numerical example for a population of aphids is investigated with the theoretical tools developed in the paper.

Copyright $(2008$ M. De La Sen. This is an open access article distributed under the Creative Commons Attribution License, which permits unrestricted use, distribution, and reproduction in any medium, provided the original work is properly cited.

\section{Introduction}

The use of mathematical models in ecology is nowadays of important research interest, [1-10] because such models lead to a more precise study of the dynamics and interactions of populations among them and with the habitat. This paper is devoted to the study of a modified version of the well-known Beverton-Holt equation in ecology which describes the population evolution of some species in a certain habitat. The associate dynamics evolves subject to an intrinsic growth rate associated with the reproduction capability and the degree of sympathy of the species with the habitat described by a so-called environment carrying capacity, $[2-6,11,12]$. The standard Beverton-Holt equation becomes the so-called generalized 
Beverton-Holt equation [12], when extended by considering in the model two extra sequences, namely, the harvesting (namely, fishing or hunting) quota, or regulation through the use of pesticides and an additive disturbance sequence. Such a disturbance sequence consists of an independent consumption sequence, which describes a predation of the species though which it gains, develops, and grows plus a net migration sequence. The migration term plays a close role to that of the independent consumption in the population evolution and it is due to potential positive/negative migration towards/from the habitat under study. In general, the joint contribution of independent consumption plus positive or negative migration (resp., immigration and emigration) towards or from the habitat could also potentially include other extra effects of correction of contribution of population in the model. Some of those effects are, for instance, unexpected accidental mortality not fixed through properly adjusting the intrinsic growth rate, loss of population due to harvesting which has not been parameterized through the harvesting quota or measuring modeling errors in general. The study in this paper adds two more sequences to the generalized Beverton-Holt equation to conform a penalty term for eventual overpopulation at intermediate sampling points. This is the main novelty of the paper related to previous literature on the subject. The penalty term corrects the population evolution so that large amounts of population translate into the decrease of the number of individuals in the future. The motivation of the use of the penalty term is clear since it is well known that large amount of individuals in a habitat makes the overall population decrease by natural regulation, for instance, due to competition for food or difficulties for nesting. The properties of boundedness and stability of the solution, existence of equilibrium points of the limit stationary version of the equation, as well as the existence of oscillatory solutions are investigated. The following basic notation is being used through the manuscript.

Notation 1.1. $\mathbf{N}$ and $\mathbf{R}$ are the sets of natural and real numbers, respectively.

$$
\begin{gathered}
\mathbf{N}_{0}:=\mathbf{N} \cup\{0\} ; \quad \mathbf{R}_{+}:=\{z \in \mathbf{R}: z>0\} ; \quad \mathbf{R}_{0+}:=\mathbf{R}_{+} \cup\{0\}=\{z \in \mathbf{R}: z \geq 0\} . \\
\bar{k}:=\{1,2, \ldots, k\} ; \quad \bar{k}^{0}:=k \cup\{0\}=\{0,1,2, \ldots, k\} .
\end{gathered}
$$

$g^{\prime}(x)$ denotes the first derivative with respect to $x$ of the differentiable real function $g(x)$. The symbols " $\wedge$ " and " $\vee$ " stand for logic conjunction and disjunction, respectively. $I_{n}$ is the identity matrix of the $n$th order. equation,

The population evolution is supposed to be governed by a modified Beverton-Holt

$$
x_{k+1}=\frac{\gamma_{k} \mu_{k} K_{k} x_{k}^{p_{k}} e^{-\alpha_{k} x_{k}} x_{k-1}}{K_{k}+\left(\mu_{k}-1\right) x_{k-1}}+d_{k} ; \quad k \in \mathbf{N}
$$

subject to initial conditions $x_{-1}>0, x_{0} \geq 0$, which is parameterized by the following real sequences.

$\gamma_{k}:=1-b_{k}, k \in \mathbf{N}$, with $b_{k} \in\left[\bar{b}_{1}, \bar{b}_{2}\right]$ being the harvesting (fishing or hunting) quota subject to $0<\bar{b}_{1} \leq \bar{b}_{2} \leq 1$. Thus, $\gamma_{k} \in\left[\bar{\gamma}_{1}, \bar{\gamma}_{2}\right], k \in \mathbf{N}$, is the harvesting quota excess subject to $0<\bar{\gamma}_{1}:=1-\bar{b}_{2} \leq \bar{\gamma}_{2}:=1-\bar{b}_{1} \leq 1$. If repopulation activity is incorporated to harvesting, then $\bar{b}_{1}<0$ when repopulation dominates harvesting so that $\bar{\gamma}_{2}>1$.

$\mu_{k} \in\left[\bar{\mu}_{1}, \bar{\mu}_{2}\right], k \in \mathbf{N}$, with $\infty>\bar{\mu}_{2} \geq \bar{\mu}_{1}>1$ is the intrinsic growth rate which is associated to the population ability for reproduction. 
$K_{k} \in\left[\bar{K}_{1}, \bar{K}_{2}\right], k \in \mathbf{N}$, with $\infty>\bar{K}_{2} \geq \bar{K}_{1}>0$, is the environment carrying capacity which is associated to the sympathetic degree, more or less favorable, of the habitat towards the species.

$d_{k} \in\left[\bar{d}_{1}, \bar{d}_{2}\right], k \in \mathbf{N}$, with $\infty>\bar{d}_{2} \geq \bar{d}_{1}>-\infty$, is the external disturbance term which refers to joint effects of independent consumption plus migration contribution, namely, immigration (i.e., positive net migration from outside towards the habitat), null, or emigration (i.e., negative net migration from the habitat to outside) including atypical mortality not included in the model by the growth rate: the disturbance. The additive disturbance contribution has the generic form $d_{k}=d_{0 k}+d_{m k}$ where $d_{0 k}$ is the independent consumption and $d_{m k}$ is the net migration contribution. The decomposition of $d_{k}=d_{0 k}+d_{m k}$ into the two separated parts $d_{0 k}$ and $d_{m k}$ is irrelevant for the modified generalized BevertonHolt equation from an analysis point of view but it is quite relevant from a biological insight point of view, as pointed out in detail by one of the reviewers. The reason is that it allows the inclusion in the same evolution equation of two quite different effects, in nature, like predation and net migration.

$p_{k} \in\left[\bar{p}_{1}, \bar{p}_{2}\right], \alpha_{k} \in\left[\bar{\alpha}_{1}, \bar{\alpha}_{2}\right]$, with $\bar{\alpha}_{k}=0 \Rightarrow \bar{p}_{k}=0, k \in \mathbf{N}$, are sequences which conform a penalty term $x_{k}^{p_{k}} e^{-\alpha_{k} x_{k}}$ to describe how the excess of population translates into a contributed tendency to its decrease at the next sampling time. The sequences are assumed to be subject to the constraints $\infty>\bar{p}_{2} \geq \bar{p}_{1} \geq 0, \infty>\bar{\alpha}_{2} \geq \bar{\alpha}_{1} \geq 0$, and $\bar{\alpha}_{1}=0 \Rightarrow \bar{p}_{1}=0$.

A particular case of (1) is obtained with $\gamma_{k}=1$ (no harvesting activity), $p_{k}=\alpha_{k}=0$ (no penalty correcting term for overpopulation control), $d_{k}=0$ (zero jointly net independent consumption plus migration) resulting in the well-known standard Beverton-Holt equation,

$$
x_{k+1}=\frac{\mu_{k} K_{k} x_{k}}{K_{k}+\left(\mu_{k}-1\right) x_{k}}, \quad k \in \mathbf{N},
$$

which is a particular version of the Hassell model $x_{k+1}=\mu_{k} x_{k} /\left(1+\left(\mu_{k}-1\right) x_{k} / K_{k}\right)^{c_{k}}, k \in \mathbf{N}$, for $c_{k}=1$ and where a double sampling period is used related to (1.2). In other words, only the recruitment and storage measurements are relevant to describe the population evolution since there is not necessary the evaluation at intermediate samples of a penalty term associated with overpopulation. Another particular case of (1.2) is obtained with $p_{k}=\alpha_{k}=0$ resulting in the so-called generalized Beverton-Holt equation,

$$
x_{k+1}=\frac{\gamma_{k} \mu_{k} K_{k} x_{k}}{K_{k}+\left(\mu_{k}-1\right) x_{k}}+d_{k}, \quad k \in \mathbf{N},
$$

also described equivalently as a two-stage evolution equation,

$$
x_{k+1}^{-}=\frac{\mu_{k} K_{k} x_{k}}{K_{k}+\left(\mu_{k}-1\right) x_{k}}, \quad x_{k+1} \equiv x_{k+1}^{+}=\gamma_{k} x_{k+1}^{-}+d_{k}, \quad k \in \mathbf{N},
$$

which includes discontinuities at sampling times due to harvesting and disturbance activities associated with impulses in the corresponding logistic continuous-time differential equation. Finally, note that the modified Beverton-Holt equation leads to the Ricker model (which is also a limiting equation of the Hassell model and that of the Beverton- Holt one if $c_{k}=1$ ):

$$
x_{k+1}=e^{\mu_{k}\left(1-x_{k} / K_{k}\right)} x_{k}, \quad k \in \mathbf{N},
$$


if $d_{k}=0$ and a harvesting quota $\gamma_{k}=\left(\left(K_{k}+\mu_{k-1} x_{k-1}\right) / K_{k}\left(\mu_{k}+x_{k-1}\right)\right) e^{\mu_{k}}, k \in \mathbf{N}$, is applied provided that $K_{k}>e^{\mu_{k}} \mu_{k-1} /\left(1+\mu_{k-1} / x_{k-1}\right), k \in \mathbf{N}$, in order to ensure that $\gamma_{k}<1$. Thus, the environment carrying capacity satisfies $\liminf _{x_{k-1} \rightarrow \infty} K_{k}>e^{\mu_{k}} \mu_{k-1}$ and $K_{k} \in\left(e^{\mu_{k}} \mu_{k-1}-\right.$ $\left.f\left(x_{k-1}\right), \infty\right), k \in \mathbf{N}$. The function $f: \mathbf{R}_{+} \rightarrow \mathbf{R}_{0+}$ is defined by $f(x)=\mu^{2} e^{\mu} /(x+\mu)$ which is decreasing on $\mathbf{R}_{+}$with $\lim _{x \rightarrow \infty} f(x)=0$ with $\mathbf{R}_{0+}:=\{z \in \mathbf{R}: z \geq 0\}$ and $\mathbf{R}_{+}:=\{z \in \mathbf{R}: z>0\}=$ $\mathbf{R}_{0+} \backslash\{0\}$. To investigate issues on the standard Beverton- Holt equation, it is useful to analyze the solution sequence of its inverse [11]. The mathematical properties of the modified generalized Beverton- Holt equation including boundedness properties, equilibrium points, and existence of oscillations are investigated. A numerical example is proposed for the study of the evolution of a population of aphids, a very resistive species of insects which causes considerable damage and reproduces according to several reproduction cycles per year $[13,14]$.

\section{Boundedness of the solutions}

There is a wide variety of works concerning boundedness of the solutions, stability, investigation of equilibrium points, and existence of oscillatory solutions in discrete recursive sequences of several types (see, e.g., [15-23]). Parallel problems are also relevant in continuous-time differential systems [16, 24-26]. To investigate those problems in the context of the modified Beverton-Holt equation (1.2), define $s_{k}:=x_{k}^{-1}, k \in \mathrm{N}$, being the solution sequence of the inverse of the modified Beverton-Holt equation provided that $x_{k} \neq 0, k \in \mathbf{N}$. Thus, one gets directly from (1.2)

$$
s_{k+1}^{-1}=\frac{\gamma_{k} \mu_{k-1} K_{k-1} \zeta_{k} s_{k-1}^{-1}}{K_{k-1}+\left(\mu_{k-1}-1\right) s_{k-1}^{-1}}+d_{k}=\frac{\gamma_{k} \mu_{k-1} K_{k-1} \zeta_{k}}{K_{k-1} s_{k-1}+\mu_{k-1}-1}+d_{k}, \quad k \in \mathbf{N},
$$

where $\zeta_{k}:=x_{k}^{p} e^{-\alpha x_{k}}, k \in \mathbf{N}$. Then,

$$
\begin{aligned}
s_{k+1} & =\frac{K_{k-1}+\left(\mu_{k-1}-1\right) x_{k-1}}{\gamma_{k} \mu_{k-1} K_{k-1} \zeta_{k} x_{k-1}+\left(K_{k-1}+\left(\mu_{k-1}-1\right) x_{k-1}\right) d_{k}}, \quad k \in \mathbf{N} \\
& =\frac{K_{k-1} s_{k-1}+\mu_{k-1}-1}{\gamma_{k} \mu_{k-1} K_{k-1} \zeta_{k}+\left(K_{k-1}+s_{k-1}+\mu_{k-1}-1\right) d_{k}}, \quad k \in \mathbf{N}
\end{aligned}
$$

which can be rewritten as

$$
\begin{aligned}
s_{k+1}= & \frac{K_{k-1}}{\gamma_{k} \mu_{k-1} K_{k-1} \zeta_{k}} s_{k-1} \\
& +\frac{\left(\mu_{k-1}-1\right) \gamma_{k} \mu_{k-1} K_{k-1} \zeta_{k}-K_{k-1} s_{k-1}\left(K_{k-1} s_{k-1}+\mu_{k-1}-1\right) d_{k}}{\gamma_{k} \mu_{k-1} K_{k-1} \zeta_{k}\left(\gamma_{k} \mu_{k-1} K_{k-1} \zeta_{k}+\left(K_{k-1} s_{k-1}+\mu_{k-1}-1\right) d_{k}\right)}, \quad k \in \mathbf{N}
\end{aligned}
$$

for the standard Beverton-Holt equation $\left(d_{k}=\alpha_{k}=p_{k} \equiv 0\right.$ implying $\zeta_{k} \equiv 1$ and $\gamma_{k} \equiv 1$ and double sampling rate; see (1.3)), (2.2)-(2.4) becomes in particular [11]

$$
s_{k+1}=\mu_{k}^{-1} s_{k}+\left(1-\mu_{k}^{-1}\right) K_{k}^{-1}, \quad k \in \mathbf{N} .
$$

The boundedness of the solutions for bounded initial conditions is a minimal requirement in order that a biological model is well posed. The subsequent result is concerned with boundedness and global Lyapunov's stability of the solution sequences of (1.2) and (2.3)(2.4). 
Theorem 2.1. The following properties hold.

(i) $\bar{d}_{1} \leq x_{k}<\infty$, for all $k \in \mathbf{N}$.

(ii) If $\bar{d}_{1}>0$, then $\left\{x_{k}\right\}_{0}^{\infty}$ and $\left\{s_{k}\right\}_{0}^{\infty}$ are positive bounded sequences. As a result, the modified Beverton-Holt equation (1.2) is globally Lyapunov's stable.

(iii) If $\bar{d}_{1} \geq 0$, then $\left\{x_{k}\right\}_{0}^{\infty}$ and $\left\{s_{k}\right\}_{0}^{\infty}$ are nonnegative sequences, the first one being bounded from above. As a result, the modified Beverton-Holt equation (1.2) is globally Lyapunov's stable. Furthermore, $\left\{s_{k}\right\}_{0}^{\infty}$ is positive with $\lim \sup _{k \rightarrow \infty} s_{k} \leq \infty$.

(iv) If $d_{k} \geq 0$ and $x_{k} \geq 0$, then $0 \leq x_{k+1}<\infty$, for all $k \in \mathbf{N}_{0}$.

Proof. (i) $x_{k} \geq d_{k} \geq \bar{d}_{1}$, for all $k \in \mathbf{N}$ follows directly from (1.2). Also, $\zeta_{k}=1$ if $\alpha_{k}=p_{k}=0$. Otherwise, $\alpha_{k} \neq 0$ so that

$$
\begin{aligned}
\zeta_{k} \leq \max _{k \in \mathbf{N}}\left(\zeta_{k}: \alpha_{k} p_{k} \neq 0\right) & =\max _{k \in \mathbf{N}}\left(\zeta\left(\frac{p_{k}}{\alpha_{k}}\right): \alpha_{k} p_{k} \neq 0\right) \\
& =\max _{k \in \mathbf{N}}\left(\left(\frac{p_{k}}{\alpha_{k} e}\right)^{p_{k}}: \alpha_{k} p_{k} \neq 0\right) \leq\left(\frac{\bar{p}_{2}}{\bar{\alpha}_{1} e}\right)^{\bar{p}_{2}}
\end{aligned}
$$

since $d \zeta(x) / d x=0$ for $\alpha \neq 0$ only at its relative maximum $x=p / \alpha$ so that $\zeta(p / \alpha)=(p / \alpha e)^{p}=$ $\max _{x \in \mathbf{R}_{0+}} \zeta(x)$ since $\zeta(0)=0$ and $\lim _{x \rightarrow \infty} \zeta(x)=0$ implying that the relative maximum is also the absolute maximum. As a result, $\zeta_{k} \leq \max \left(1,\left(\bar{p}_{2} / \bar{\alpha}_{1} e\right)^{\bar{p}_{2}}\right)<\infty$, for all $k \in \mathbf{N}$. Then, from (1.2)

$$
x_{k+1} \leq \frac{\bar{\gamma}_{2} \bar{\mu}_{2} \bar{K}_{2} x_{k-1}}{\bar{K}_{1}+\left(\bar{\mu}_{1}-1\right) x_{k-1}} \max \left(1,\left(\frac{\bar{p}_{2}}{\bar{\alpha}_{1} e}\right)^{\bar{p}_{2}}\right)+\bar{d}_{2} \leq \frac{\bar{\gamma}_{2} \bar{\mu}_{2} \bar{K}_{2} \max \left(1,\left(\bar{p}_{2} / \bar{\alpha}_{1} e\right)^{\bar{p}_{2}}\right)}{\bar{\mu}_{1}-1}+\bar{d}_{2}<\infty,
$$

for all $k \in \mathbf{N}$ and (i) has been proved.

If $\bar{d}_{1}>0$, then (i) implies $0<\bar{d}_{1} \leq x_{k}<\infty$ and $0<s_{k} \leq \bar{d}_{1}^{-1}<\infty$, for all $k \in \mathbf{N}$ and (ii) has been proved.

If $\bar{d}_{1} \geq 0$, then (i) implies $0 \leq x_{k}<\infty$ and $0<s_{k} \leq \infty$, for all $k \in \mathbf{N}$ and, thus, $\liminf \operatorname{in}_{k \rightarrow \infty} x_{k} \geq 0, \limsup _{k \rightarrow \infty} x_{k}<\infty, \liminf _{k \rightarrow \infty} s_{k}>0$, and limsup $\sup _{k \rightarrow \infty} s_{k} \leq \infty$. Property (iii) has been proved. Property (iv) follows directly from (1.2).

For formulation coherency, the population cannot be negative at any sample. This idea motivates the following axiom and simple-related assertions which will be then useful for some mathematical proofs concerning the case of the eventual negativity of the joint disturbance contribution.

Axiom 2.2. $x_{k} \geq 0$, for all $k \in \mathbf{N}$.

Assertion 2.3. $x_{k}=0 \Rightarrow d_{k} \geq 0$ for any $k \in \mathbf{N}$.

Assertion 2.4. $\bar{d}_{2}<0 \Rightarrow x_{k}>0$, for all $k \in \mathbf{N}$.

Assertion 2.5. $d_{k}<0 \Rightarrow \zeta_{k}>0$ for any $k \in \mathbf{N}$. 
Assertion 2.6. $\zeta_{k}=0$ only if $x_{k}=0$ and $d_{k} \geq 0$ for any $k \in \mathbf{N}$.

Proofs of Assertions 2.3-2.6. Assertion 2.4 is equivalent to prove that its contrapositive proposition $x_{k} \leq 0$ for some $k \in \mathbf{N} \Rightarrow \bar{d}_{2} \geq 0$ is true. Assume that $x_{k} \leq 0$ for some $k \in \mathbf{N}$. Then, Axiom 2.2 implies that $x_{k}=0$ so that $\zeta_{k}=0$ and then $x_{k+1}=d_{k} \geq 0$ from (1.2) and Axiom 2.2 so that Assertion 2.3 is proved. Also, $x_{k} \leq 0 \Rightarrow \bar{d}_{2} \geq \max _{j \in \mathbf{N}}\left(d_{j}\right) \geq d_{k} \geq 0 \Leftrightarrow \bar{d}_{2}<0 \Rightarrow x_{k}>$ 0 , for all $k \in \mathbf{N}$ what proves Assertion 2.4. Assertion 2.5 follows since Axiom $2.2 \wedge d_{k}<0$

$$
\begin{aligned}
\Longrightarrow \zeta_{k} & =\frac{\left(K_{k-1}+\left(\mu_{k-1}-1\right) x_{k-1}\right)\left|d_{k}\right|}{\gamma_{k} \mu_{k-1} K_{k-1} x_{k-1}} \quad\left(\Longleftrightarrow x_{k+1} \geq 0\right) \\
& \geq \frac{\left(\bar{\mu}_{1}-1\right)\left|d_{k}\right|}{\bar{\gamma}_{2} \bar{\mu}_{2} \bar{K}_{2}}+\frac{\bar{K}_{1}\left|d_{k}\right|}{\bar{\gamma}_{2} \bar{\mu}_{2} \bar{K}_{k-1}} \geq \frac{\left(\bar{\mu}_{1}-1\right)\left|d_{k}\right|}{\bar{\gamma}_{2} \bar{\mu}_{2} \bar{K}_{2}}>0 \quad \text { for any } k \in \mathbf{N} .
\end{aligned}
$$

Assertion 2.6 follows since for any $k \in \mathbf{N}, \zeta_{k}=0 \Rightarrow x_{k}=0 \vee x_{k}=\infty . x_{k}=\infty$ is impossible from Theorem 2.1(i) so that for any $k \in \mathbf{N}, \zeta_{k}=0 \Rightarrow x_{k}=0$. If $x_{k}=0$, then $x_{k+1} \geq 0$ from Axiom 2.2 so that $d_{k} \geq 0$ which is Assertion 2.3.

The extinction of the standard Beverton-Holt equation is investigated in [27] when the intrinsic growth rate is less then unity. Note that eventual extinction is also admitted by Axiom 2.2 for intrinsic growth rate exceeding unity and negative disturbance contribution. This situation includes as particular cases that of local emigration plus zero-independent consumption and that of negative-independent consumption with no net migration contribution. However, the axiom prevents against eventual negative levels of population which is not physically possible.

Remark 2.7. Note that Assertion 2.5 together with an intermediate result in the proof of Theorem 2.1 implies $d_{k}<0 \Rightarrow \infty>\max \left(1,\left(\bar{p}_{2} / \bar{\alpha}_{1} e\right)^{\bar{p}_{2}}\right) \geq \zeta_{k}>0$ for any $k \in \mathbf{N}$,

$$
d_{k} \geq 0 \Longrightarrow \infty>\max \left(1,\left(\frac{\bar{p}_{2}}{\bar{\alpha}_{1} e}\right)^{\bar{p}_{2}}\right) \geq \zeta_{k} \geq 0 \quad \text { for any } k \in \mathbf{N}
$$

Then, $\infty>\zeta_{k} \geq 0$, for all $k \in \mathbf{N}$, and $\infty>\limsup _{k \rightarrow \infty} \zeta_{k} \geq \liminf \inf _{k \rightarrow \infty} \zeta_{k} \geq 0$. Also, if $\bar{d}_{2}<0$ or if $d_{k}<0$ (although a nonnegative upper-bound be assumed), for all $k \in \mathbf{N}$, then $\infty>$ $\limsup _{k \rightarrow \infty} \zeta_{k} \geq \liminf _{k \rightarrow \infty} \zeta_{k}>0$.

Now, Axiom 2.2 and Assertions 2.3-2.6 extend the results of Theorem 2.1 concerning positivity of the solutions and further stability results as follows.

Theorem 2.8. The following properties hold under Axiom 2.2.

(i) $\left\{x_{k}\right\}_{0}^{\infty}$ and $\left\{s_{k}\right\}_{0}^{\infty}$ are nonnegative sequences which are bounded and positive and bounded from below, respectively, independently of the disturbance contribution. As a result, the modified Beverton-Holt equation (1.2) is globally Lyapunov's stable. Furthermore, if $d_{k}>$ 0 , for all $k \in \mathbf{N}$, both sequences are positive and bounded. 
(ii) Define $\rho_{k}:=1 / \gamma_{k} \mu_{k-1} \zeta_{k}, \delta_{k}:=K_{k-1} s_{k-1}+\mu_{k-1}-1=\left(K_{k-1}+\left(\mu_{k-1}-1\right) x_{k-1}\right) / x_{k-1}, \eta_{k}:=$ $v_{k}-\omega_{k}, v_{k}:=\left(\mu_{k-1}-1\right) /\left(\rho_{k}^{-1}+\delta_{k} d_{k}\right)$, and $\omega_{k}:=K_{k-1} s_{k-1} \delta_{k} d_{k} / \rho_{k}^{-1}\left(\rho_{k}^{-1}+\delta_{k} d_{k}\right)$. If $d_{k}>0$, for all $k \in \mathbf{N}$, then either

$0<\prod_{i=0}^{k}\left[\rho_{i}\right]<\infty ; \quad \forall k \in \mathbf{N}\left(\right.$ then $\left.0<\liminf _{k \rightarrow \infty} \prod_{i=0}^{k}\left[\rho_{i}\right] \leq \limsup _{k \rightarrow \infty} \prod_{i=0}^{k}\left[\rho_{i}\right]<\infty\right)$

$\wedge$

$0 \leq \sum_{i=0}^{k-1} \prod_{j=i+1}^{k-1}\left[\rho_{j}\right] \eta_{i}<\infty\left(\Longrightarrow 0 \leq \sum_{i=0}^{k-1} \prod_{j=i+1}^{k-1}\left[\rho_{j}\right] v_{i} \leq \infty \wedge 0 \leq \sum_{i=0}^{k-1} \prod_{j=i+1}^{k-1}\left[\rho_{j}\right] \omega_{i} \leq \infty\right), \quad \forall k \in \mathbf{N}$,

or $\lim _{k \rightarrow \infty} \prod_{i=0}^{k}\left[\rho_{i}\right]=-\lim _{k \rightarrow \infty} \sum_{i=0}^{k-1} \prod_{j=i+1}^{k-1}\left[\rho_{j}\right] \eta_{i}=\infty \Longrightarrow\left(\lim _{k \rightarrow \infty} \sum_{i=0}^{k-1} \prod_{j=i+1}^{k-1}\left[\rho_{j}\right] \omega_{i}=\infty\right)$.

(iii) If $d_{k} \leq 0$, for all $k \in \mathbf{N}$, then either

$0<\prod_{i=0}^{k}\left[\rho_{i}\right] \leq \infty ; \quad \forall k \in \mathbf{N}$

$\wedge$

$0 \leq \sum_{i=0}^{k-1} \prod_{j=i+1}^{k-1}\left[\rho_{j}\right] \eta_{i} \leq \infty\left(\Longrightarrow 0 \leq \sum_{i=0}^{k-1} \prod_{j=i+1}^{k-1}\left[\rho_{j}\right] v_{i} \leq \infty \wedge 0 \leq \sum_{i=0}^{k-1} \prod_{j=i+1}^{k-1}\left[\rho_{j}\right] \omega_{i} \leq \infty\right), \quad \forall k \in \mathbf{N}$,

or $\lim _{k \rightarrow \infty} \prod_{i=0}^{k}\left[\rho_{i}\right]=\lim _{k \rightarrow \infty} \sum_{i=0}^{k-1} \prod_{j=i+1}^{k-1}\left[\rho_{j}\right] \eta_{i}=\infty \Longrightarrow\left(\lim _{k \rightarrow \infty} \sum_{i=0}^{k-1} \prod_{j=i+1}^{k-1}\left[\rho_{j}\right] \omega_{i}=\infty\right)$.

Proof. (i) Note from (1.2), Axiom 2.2, and Assertion 2.5 that, for $d_{k}<0$,

$$
\zeta_{k}>0 \wedge\left|d_{k}\right|=-d_{k} \leq \frac{\gamma_{k-1} \mu_{k-1} \mu_{k-1} \zeta_{k}}{K_{k-1}+\left(\mu_{k-1}-1\right) x_{k-1}}=\frac{\gamma_{k-1} \mu_{k-1} \mu_{k-1} \zeta_{k} s_{k-1}}{K_{k-1} s_{k-1}+\left(\mu_{k-1}-1\right)}>0
$$

so that from (2.4)

$$
\begin{aligned}
s_{k+1} \geq & \frac{K_{k-1}}{\gamma_{k} \mu_{k-1} K_{k-1} \zeta_{k}} s_{k-1} \\
& +\frac{\left(\mu_{k-1}-1\right) \gamma_{k} \mu_{k-1} K_{k-1} \zeta_{k}+K_{k-1} s_{k-1}\left(K_{k-1} s_{k-1}+\mu_{k-1}-1\right)\left|d_{k}\right|}{\gamma_{k} \mu_{k-1} K_{k-1} \zeta_{k}\left(\gamma_{k} \mu_{k-1} K_{k-1} \zeta_{k}-\left(K_{k-1} s_{k-1}+\mu_{k-1}-1\right)\left|d_{k}\right|\right)} \\
\geq & \frac{K_{k-1}}{\gamma_{k} \mu_{k-1} K_{k-1} \zeta_{k}} s_{k-1}>0 \Longleftrightarrow 0 \leq x_{k+1}<\infty
\end{aligned}
$$

for any $k \in \mathbf{N}_{0}$ if $d_{k}<0$. On the other hand, $d_{k} \geq 0 \Rightarrow 0 \leq x_{k+1}<\infty$ and $0<s_{k+1} \leq \infty$ for any $k \in \mathbf{N}_{0}$ from Theorem 2.1(iv). Furthermore, $d_{k}>0 \Rightarrow 0<x_{k+1}<\infty$ and $0<s_{k+1}<\infty$ for any $k \in \mathbf{N}_{0}$ from Theorem 2.1(ii). As a result, Property (i) has been fully proved. 
(ii) Recursive direct calculations from (2.4) yield taking (i) into account irrespective of the value of the disturbance contribution:

$$
0<s_{k}=\prod_{i=0}^{k}\left[\rho_{i}\right] s_{0}+\sum_{i=0}^{k-1} \prod_{j=i+1}^{k-1}\left[\rho_{j}\right] \eta_{i}=\prod_{i=0}^{k}\left[\rho_{i}\right] s_{0}+\sum_{i=0}^{k-1} \prod_{j=i+1}^{k-1}\left[\rho_{j}\right]\left(v_{i}-\omega_{i}\right) \leq \infty, \quad \forall k \in \mathbf{N}
$$

and, furthermore, if $d_{k}>0$; for all $k \in \mathbf{N}$ then

$$
0<s_{k}=\prod_{i=0}^{k}\left[\rho_{i}\right] s_{0}+\sum_{i=0}^{k-1} \prod_{j=i+1}^{k-1}\left[\rho_{j}\right] \eta_{i}=\prod_{i=0}^{k}\left[\rho_{i}\right] s_{0}+\sum_{i=0}^{k-1} \prod_{j=i+1}^{k-1}\left[\rho_{j}\right]\left(v_{i}-\omega_{i}\right)<\infty, \quad \forall k \in \mathbf{N}
$$

since $\left\{x_{k}\right\}_{0}^{\infty}$ is positive and bounded from Theorem 2.1. Thus, the first two constraints linked via logic conjunction follow directly from (2.14) since either $\prod_{i=0}^{k}\left[\rho_{i}\right]<$ $\infty$, for all $k \in \mathbf{N}$, so that $\left|\sum_{i=0}^{k-1} \prod_{j=i+1}^{k-1}\left[\rho_{j}\right] \eta_{i}\right|<\infty$, for all $k \in \mathbf{N}$, or $\lim _{k \rightarrow \infty} \prod_{i=0}^{k}\left[\rho_{i}\right]=$ $-\lim _{k \rightarrow \infty}\left(\sum_{i=0}^{k-1} \prod_{j=i+1}^{k-1}\left[\rho_{j}\right] \eta_{i}\right)=\infty$, or $\prod_{i=0}^{k}\left[\rho_{i}\right]=-\left(\sum_{i=0}^{k-1} \prod_{j=i+1}^{k-1}\left[\rho_{j}\right] \eta_{i}\right)=\infty$ for finite $k \in \mathbf{N}$. However, from the equivalent contrapositive logic proposition to Assertion 2.6, $x_{k}>0$ (which holds from Theorem 2.1, since $\left.d_{k}>0\right) \Rightarrow \zeta_{k}>0$ which implies $\max \left(\prod_{i=0}^{k}\left[\rho_{i}\right],\left|\sum_{i=0}^{k-1} \prod_{j=i+1}^{k-1}\left[\rho_{j}\right] \eta_{i}\right|\right)<\infty$ from $(2.15)$ and then $\prod_{i=0}^{k}\left[\rho_{i}\right]=-\left(\sum_{i=0}^{k-1} \prod_{j=i+1}^{k-1}\left[\rho_{j}\right] \eta_{i}\right)=$ $\infty$ for finite $k \in \mathbf{N}$ is impossible. Property (ii) has been proved.

(iii) It is proved using similar techniques as those used in the proof of (ii) by noting that $\left|d_{k}\right|$ is upper bounded with the bound of (2.12) for $d_{k} \leq 0$ and $\delta_{k}>0, \rho_{k}>0, \zeta_{k} \geq 0, \infty \geq \eta_{k} \geq$ 0 , for all $k \in \mathbf{N}$. The detailed proof is omitted.

Remark 2.9. Note that the use of the contrapositive logic proposition to Assertion 2.6 is not feasible, as invoked in the last part of the proof of Theorem 2.8(ii), in order to prove some close result concerned with Theorem 2.8(iii). This follows since $d_{k}<0 \Rightarrow \zeta_{k}>0$, for all $k \in \mathbf{N}$ from Assertion 2.5.

\section{Equilibrium points}

Assume that $\lim _{k \rightarrow \infty} K_{k}=K, \lim _{k \rightarrow \infty} \mu_{k}=\mu, \lim _{k \rightarrow \infty} \gamma_{k}=\gamma, \lim _{k \rightarrow \infty} \alpha_{k}=\gamma, \lim _{k \rightarrow \infty} p_{k}=$ $\gamma, \lim _{k \rightarrow \infty} d_{k}=d$. It is well known that both the standard Beveron-Holt equation, driven by two parameters, and the generalized one, driven by four parameters, have equilibrium points. In particular, the equilibrium point under nonextinction condition of the standard Beverton-Holt equation is the carrying capacity of the environment; see [2-6, 11-13, 27, 28]. The following result holds, which is concerned with the existence of equilibrium points in the limit equation (1.2), that is, the stationary solutions $\lim _{k \rightarrow \infty} x_{k}=\bar{x} \geq 0$ of (1.2) as the parameters converge to those finite limits.

Theorem 3.1. The following properties hold.

(i) If $d>0$, then it exists a unique equilibrium point $x=\bar{x}$ on $\mathbf{R}_{0+}$ which is, in addition, positive being subject either to $x_{M}>\bar{x} \geq x_{b}$, which requires $\alpha \in[0,(p+1) / d)$ as a necessary condition, or $x_{M} \leq \bar{x}<x_{b}$, which requires $\alpha \geq(p+1) / d$. 
(ii) If $d<0$, then an equilibrium point $x=\bar{x}$ on $\mathbf{R}_{0+}$ can exist only if the following two necessary conditions hold:

(i) $|d| \leq(1 / K)((p+1) / \alpha e)^{p+1}$;

(ii) $K+(\mu-1)|d|<\gamma \mu K(p+1) x^{p} e^{-\alpha x}-\left(\gamma \mu K \alpha x^{p} e^{-\alpha x}+2(\mu-1)\right) x$ for $x \in X_{M} \subset\left[0, x_{M}\right)$. If there exists such an equilibrium point, then at most two distinct equilibrium points satisfying $0<\bar{x}_{1} \leq x_{M} \leq \bar{x}_{2}$ can exist.

(iii) If $d=0$, then $\bar{x}_{1}=0$ is an equilibrium point of (1.2). Another equilibrium point might exist only if $K\left[\gamma \mu K p x^{p} e^{-\alpha x}(p+1-\alpha x)-1\right]>2(\mu-1) x$ on some interval $X_{M} \subset\left[0, x_{M}\right)$.

Proof. The equilibrium points of (1.2), if any, are the (real) values of $x \in \mathbf{R}_{0+}$ such that

$$
f(x):=(\mu-1) x^{2}+(K-d(\mu-1)) x-d K=g(x):=\gamma \mu K \zeta(x) x .
$$

Note that $f: \mathbf{R} \rightarrow \mathbf{R}$ is a convex parabola satisfying $f(0)=-K d$ whose zeros are

$$
x_{a, b}=\frac{(\mu-1) d-K \mp\left((K-(\mu-1) d)^{2}+4(\mu-1) K d\right)^{1 / 2}}{2(\mu-1)}=\left\{\begin{array}{l}
-\frac{K}{\mu-1}<0 \\
d
\end{array}\right.
$$

since $\left((K-(\mu-1) d)^{2}+4(\mu-1) K d\right)^{1 / 2}=K+(\mu-1) d$. Also, $g: \mathbf{R} \rightarrow \mathbf{R}$ satisfies $g(0)=0, g(\infty)=$ $\lim _{k \rightarrow \infty} g(x)=0$ and has a relative maximum, which is also the absolute maximum in $\left.\mathbf{R}_{0_{+}}\right)$at $x_{M}=(p+1) / \alpha$ which is $g_{M}=((p+1) / \alpha e)^{p+1}=\max \left(g(x): x \in \mathbf{R}_{0+}\right)$. Also, $g(x)$ is increasing on $x \in\left[0, x_{M}\right)$ and decreasing on $x \in\left[x_{M}, \infty\right)$. Thus, the equilibrium points $x=\bar{x}$, if any, related to $x_{b}$ and $x_{M}$ satisfy the subsequent constraints.

(a) If $d>0$, then $f(0)=-K d<g(0)=0$. Thus, there is a unique equilibrium point $x=\bar{x}$ (i.e., $f(\bar{x})=g(\bar{x}))$ on $\mathbf{R}_{0+}$ which is, in addition, positive which is subject either to $x_{M}>\bar{x} \geq x_{b}$, which requires $\alpha \in[0,(p+1) / d)$ as a necessary condition, or $x_{M} \leq \bar{x}<x_{b}$, which requires $\alpha \geq(p+1) / d$.

(b) If $d<0$, then $f(0)=K|d|>g(0)=0$ and $x_{a} \leq x_{b}<0$. Thus, an equilibrium point $x=\bar{x}$ (i.e., $f(\bar{x})=g(\bar{x})$ ) on $\mathbf{R}_{0+}$ can exist only if the following two necessary conditions hold:

(i) $|d| \leq(1 / K)((p+1) / \alpha e)^{p+1}$ implying $f(0) \leq g_{M}$ since $f(x)$ is increasing on $\left[x_{b}, \infty\right)$ so that it is also increasing on $\mathbf{R}_{0+}$ since $\mathbf{R}_{0+} \subset\left[x_{b}, \infty\right)$;

(ii) $K+(\mu-1)|d|<\gamma \mu K(p+1) x^{p} e^{-\alpha x}-\left(\gamma \mu K \alpha x^{p} e^{-\alpha x}+2(\mu-1)\right) x$ for $x \in X_{M} \subset\left[0, x_{M}\right)$ since $f(0)>g(0)$ and $f(x)$ being increasing on $[0, \infty)$ implies that solutions to $f(x)=g(x)$ might exist only if $f(0) \leq g_{M}$ and the continuous function $h(x):=$ $g(x)-f(x)$ is monotonically strictly increasing (i.e., $h^{\prime}(x)=g^{\prime}(x)-f^{\prime}(x)>0$ on some interval $X_{M} \subset\left[0, x_{M}\right)$. 
If there exists such an equilibrium point, then it can exist at most two distinct equilibrium points satisfying $0<\bar{x}_{1} \leq x_{M} \leq \bar{x}_{2}$.

(c) If $d=0$, then $f(0)=g(0)=0$ so that $\bar{x}_{1}=0$ is an equilibrium point of (1.2). Another equilibrium point might exist only if $K\left[\gamma \mu K p x^{p} e^{-\alpha x}(p+1-\alpha x)-1\right]>2(\mu-1) x$ in order that $h(x):=g(x)-f(x)$ be monotonically strictly increasing on some interval $X_{M} \subset\left[0, x_{M}\right)$.

Remark 3.2. Particular cases of equilibrium points of interest are the following.

(i) The case $p=\alpha=d=0$. Thus, the constraint (3.1) becomes $f(x)=(\mu-1) x^{2}+K x=$ $g(x)=\gamma \mu K x$ which yields an equilibrium point at $\bar{x}=K(\gamma \mu-1) /(\mu-1)$, provided that $1>\gamma>\mu^{-1}$. If $\gamma=1$, one obtains the equilibrium point of the standard Beverton-Holt equation $\bar{x}=K$.

(ii) The case $p=\alpha=0$; $d>0$. Thus, $x=\gamma \mu K x /(K+(\mu-1) x)$ holds for $x=\bar{x}_{1}=$ $K(\gamma \mu-1) /(\mu-1)$ provided that $1>\gamma>\mu^{-1}$, and which implies that $\lim _{k \rightarrow \infty} x_{k}^{-}=\bar{x}_{1}$. Also, $x=\gamma x+d$ holds for $x=\bar{x}_{2}=d /(1-\gamma)>0$ since $d>0$ and $1>\gamma>0$, which implies that $\lim _{k \rightarrow \infty} x_{k}=\lim _{k \rightarrow \infty} x_{k}^{+}=\bar{x}_{2}$. Then, $\bar{x}=\bar{x}_{1}=\bar{x}_{2}$ is an equilibrium point of (1.2), provided that $1>\gamma>\mu^{-1}$, implying that $\lim _{k \rightarrow \infty} x_{k}=\lim _{k \rightarrow \infty} x_{k}^{+}=\lim _{k \rightarrow \infty} x_{k}^{-}=\bar{x}$ if $\bar{x}=K(\gamma \mu-1) /(\mu-1)=d /(1-\gamma)$ if $d=(1-\gamma)(\gamma \mu-1) K /(\mu-1)$.

Lower and upper bounds for the equilibrium points of the limit stationary equation (1.2) are investigated in the subsequent result. The use of those bounds is important when the exact equilibrium point cannot be calculated by imprecise knowledge of the model parameterization or computational difficulties.

Theorem 3.3. The following properties hold.

(i) The equilibrium points $\bar{x}_{1,2}$ of the limit stationary modified Beverton-Holt equation (1.2) satisfy the following properties:

$$
0 \leq d=x_{22} \leq \bar{x}_{2} \leq x_{12} \leq \sqrt{\frac{d K}{\mu-1}}
$$

provided that $K>d(\mu-1)$ so that $\bar{x}_{2}=0$ if and only if $d=0$. If $d<0$ and has a sufficiently small modulus, then $0>-|d|=x_{22} \leq \bar{x}_{2} \leq x_{12}<0$. Also,

$$
\begin{gathered}
-\sqrt{\frac{d K}{\mu-1}} \geq x_{11} \leq \bar{x}_{1} \leq x_{21}=-\frac{K}{(\mu-1)}<0 \quad \text { if } d \geq 0, \\
\min \left(x_{11}, x_{12}\right) \leq \bar{x}_{1} \leq \max \left(-\frac{K}{(\mu-1)},-|d|\right)<0, \quad \text { if } d<0 .
\end{gathered}
$$

(ii) Define the real functions $\psi(x)=\gamma K \mu x /(K+(\mu-1) x)$ and $\zeta(x)=x^{p} e^{-\alpha x}$. Then, any equilibrium point $\bar{x}$ of the limit stationary Beverton-Holt equation (1.2) is locally asymptotically stable if the two constraints below jointly hold:

$$
1+\zeta(\bar{x}) \psi^{\prime}(\bar{x})>0, \quad\left|\psi(\bar{x}) \zeta^{\prime}(\bar{x})\right|+\zeta(\bar{x}) \psi^{\prime}(\bar{x})<1 .
$$


Proof. Note from (3.1) that

$$
\begin{aligned}
h_{2}(x) & :=(\mu-1) x^{2}+(K-d(\mu-1)) x-d K \\
& \geq h(x):=f(x)-g(x)=(\mu-1) x^{2}+(K-d(\mu-1)-\gamma \mu K \zeta(x)) x-d K \\
& \geq h_{1}(x):=(\mu-1) x^{2}+\left(K-d(\mu-1)-\gamma \mu K\left(\frac{p}{\alpha e}\right)^{p}\right) x-d K
\end{aligned}
$$

since $\zeta(x) \leq(p / \alpha e)^{p}$, for all $x \in \mathbf{R}_{0+}$. From (3.1), the equilibrium points are the nonnegative real solutions to $h(x)=0$, if any. The zeros of the convex parabola $h_{1}(x)$ are

$$
\begin{aligned}
x_{11} & =\frac{d(\mu-1)+\gamma \mu K(p / \alpha e)^{p}-K-\left(\left(d(\mu-1)+\gamma \mu K(p / \alpha e)^{p}-K\right)^{2}+4(\mu-1) d K\right)^{1 / 2}}{2(\mu-1)} \\
& \leq-\sqrt{\frac{d K}{\mu-1}} \leq 0
\end{aligned}
$$

if $d \geq 0, x_{11}>0$ if $d<0$ of sufficiently small modulus, and $x_{11}<0$ if $d<0$ of sufficiently large modulus

$$
\begin{aligned}
x_{12} & =\frac{d(\mu-1)+\gamma \mu K(p / \alpha e)^{p}-K+\left(\left(d(\mu-1)+\gamma \mu K(p / \alpha e)^{p}-K\right)^{2}+4(\mu-1) d K\right)^{1 / 2}}{2(\mu-1)} \\
& \leq \sqrt{\frac{d K}{\mu-1}}
\end{aligned}
$$

if $d \geq 0$ and $x_{12}<0$ if $d<0$ of sufficiently small modulus or complex if $d<0$ of sufficiently large modulus.

The zeros of the convex parabola $h_{2}(x)$ are

$$
\begin{aligned}
& x_{21}=\frac{d(\mu-1)-K-\left((d(\mu-1)+K)^{2}\right)^{1 / 2}}{2(\mu-1)}=-\frac{K}{(\mu-1)}<0, \\
& x_{22}=\frac{d(\mu-1)-K+\left((d(\mu-1)+K)^{2}\right)^{1 / 2}}{2(\mu-1)}=d .
\end{aligned}
$$

In view of (3.6), since $h_{1}(x), h_{2}(x)$, and $h(x)$ are convex parabolas, subject to (3.6), the zeros $\bar{x}_{1,2}$ of $h(x)$, which are the equilibrium points of the limit stationary (1.2), satisfy

$$
\begin{aligned}
0 & \leq d=x_{22} \leq \bar{x}_{2} \leq x_{12} \\
& =\frac{d(\mu-1)+\gamma \mu K(p / \alpha e)^{p}-K+\left(\left(\gamma \mu K(p / \alpha e)^{p}+d(\mu-1)-K\right)^{2}+4(\mu-1) d K\right)^{1 / 2}}{2(\mu-1)} \\
& \leq \sqrt{\frac{d K}{\mu-1}}
\end{aligned}
$$


provided that $d \geq 0$ and $K>d(\mu-1)$ so that $\bar{x}_{2}=0$ if and only if $d=0$. If $d<0$ of sufficiently small modulus, then

$$
\begin{aligned}
& 0>-|d|=x_{22} \leq \bar{x}_{2} \leq x_{12} \\
& =\frac{\gamma \mu K(p / \alpha e)^{p}-|d|(\mu-1)-K+\left(\left(\gamma \mu K(p / \alpha e)^{p}-|d|(\mu-1)-K\right)^{2}-4(\mu-1)|d| K\right)^{1 / 2}}{2(\mu-1)}<0, \\
& \quad-\sqrt{\frac{d K}{\mu-1}} \geq x_{11} \leq \bar{x}_{1} \leq x_{21}=-\frac{K}{(\mu-1)}<0 \quad \text { if } d \geq 0, \\
& \min \left(x_{11}, x_{12}\right) \leq \bar{x}_{1} \leq \max \left(-\frac{K}{(\mu-1)},-|d|\right)<0 \quad \text { if } d<0,
\end{aligned}
$$

so that Property (i) has been proved. To prove Property (ii), first note that the modified limit Beverton-Holt equation (1.2) may be written more compactly as

$$
x_{k+1}-d=\psi\left(x_{k-1}\right) \zeta\left(x_{k}\right),
$$

where

$$
\psi(x)=\frac{\gamma K \mu x}{K+(\mu-1) x}, \quad \zeta(x)=x^{p} e^{-\alpha x}
$$

which implies

$$
\psi^{\prime}(x)=\frac{\gamma K^{2} \mu}{(K+(\mu-1) x)^{2}} ; \quad \zeta^{\prime}(x)=x^{p-1} e^{-\alpha x}(p-\alpha x) .
$$

The equilibrium points $\bar{x}$, if any, satisfy $\bar{x}-d=\lim _{k \rightarrow \infty} x_{k}-d=\psi(\bar{x}) \zeta(\bar{x})$. The linearized dynamics of (3.12) about the equilibrium points are [19]

$$
z_{k+1}=\zeta(\bar{x}) \psi^{\prime}(\bar{x}) z_{k-1}+\psi(\bar{x}) \zeta^{\prime}(\bar{x}) z_{k}
$$

whose associated characteristic equation in the complex indeterminate $\mathcal{\lambda}$ is

$$
\lambda^{2}-\psi(\bar{x}) \zeta^{\prime}(\bar{x}) \lambda-\zeta(\bar{x}) \psi^{\prime}(\bar{x})=0
$$

so that the equilibrium point is locally asymptotically stable if both zeros of (3.16) have modulus less than unity, namely,

$$
1>-\zeta(\bar{x}) \psi^{\prime}(\bar{x})>\left|\psi(\bar{x}) \zeta^{\prime}(\bar{x})\right|-1
$$

or, equivalently,

$$
1+\zeta(\bar{x}) \psi^{\prime}(\bar{x})>0 ; \quad\left|\psi(\bar{x}) \zeta^{\prime}(\bar{x})\right|+\zeta(\bar{x}) \psi^{\prime}(\bar{x})<1
$$


For $d \geq 0$, since, $x=\bar{x}_{2} \leq x_{12}$, it follows that $\psi(\bar{x}) \leq \gamma \mu \bar{x} \leq \gamma \mu \bar{x}_{12} \leq \gamma \mu \sqrt{d K /(\mu-1)}$ and also that $\psi(\bar{x}) \leq \gamma K \mu /(\mu-1)$, for all $x \in \mathbf{R}_{0+}$ directly from (3.13) so that

$$
\begin{gathered}
\psi(\bar{x}) \leq \min \left(\frac{\gamma K \mu}{(\mu-1)}, \gamma \mu \sqrt{\frac{d K}{\mu-1}}\right) \\
d^{p} e^{-\alpha \sqrt{d K /(\mu-1)}} \leq \zeta(\bar{x}) \leq \max \left(\zeta(x): x \in \mathbf{R}_{0+}\right)=\left(\frac{p}{\alpha e}\right)^{p}
\end{gathered}
$$

if $d \geq 0$ from the constraint. From that constraint and $0 \leq d=x_{22} \leq \bar{x}_{2} \leq x_{12}$ and (3.13)-(3.14),

$$
\frac{\gamma K^{2} \mu}{(K+\sqrt{d K(\mu-1)})^{2}} \leq \psi^{\prime}(\bar{x}) \leq \gamma \mu \min \left(1, \frac{K^{2}}{(\mu-1)^{2} d^{2}}\right) .
$$

$\zeta^{\prime}(x)$ defined in (3.14) has a derivative $(d / d x) \zeta^{\prime}(x)=x^{p-2} e^{-\alpha x}[(p-1) p-(p+1) \alpha x]$ which has a relative extremum at $x=(p-1) p /(p+1) \alpha$. Thus, taking into account also (3.14) and $0 \leq d=x_{22} \leq \bar{x}_{2} \leq x_{12}$ for $d \geq 0$, one gets

$$
\begin{aligned}
& d^{p-1} e^{-\alpha \sqrt{d K /(\mu-1)}}\left(p-\alpha \sqrt{\frac{d K}{\mu-1}}\right) \\
& \quad \leq \zeta^{\prime}(\bar{x}) \\
& \quad \leq \min \left(\left(\frac{d K}{\mu-1}\right)^{(p-1) / 2} e^{-d \alpha}(p-\alpha d), \frac{2 p}{p+1}\left(\frac{(p-1) p}{(p+1) \alpha}\right)^{p-1} e^{(p-1) p /(p+1)}\right) .
\end{aligned}
$$

Thus, from (3.18), sufficient conditions for the equilibrium point $\bar{x}=\bar{x}_{2}$ to be locally stable if $p \geq \alpha d$ are from (3.19) to (3.21):

$$
\begin{aligned}
& d^{p} e^{-\alpha \sqrt{d K /(\mu-1)}} \frac{\gamma K^{2} \mu}{(K+\sqrt{d K(\mu-1)})^{2}}>-1, \\
& \left|\min \left(\frac{\gamma K \mu}{(\mu-1)}, \gamma \mu \sqrt{\frac{d K}{\mu-1}}\right) \min \left(\left(\frac{d K}{\mu-1}\right)^{(p-1) / 2} e^{-d \alpha}(p-\alpha d), \frac{2 p}{p+1}\left(\frac{(p-1) p}{(p+1) \alpha}\right)^{p-1} e^{(p-1) p /(p+1)}\right)\right| \\
& \quad+\gamma \mu\left(\frac{p}{\alpha e}\right)^{p} \min \left(1, \frac{K^{2}}{(\mu-1)^{2} d^{2}}\right)<1 .
\end{aligned}
$$

Theorem 3.1(i)-(ii) establish that there is a unique equilibrium point on $\mathbf{R}_{+}$for $d>0$ of the limit stationary equation (1.2) and that at most two equilibrium points might exist on $\mathbf{R}_{+}$for $d<0$ being of small absolute value. Those results are obtained by investigating the zeros of the upper-bounding and lower-bounding parabolas to $h(x)$. The subsequent result gives explicit conditions of the existence of two equilibrium points in such a situation provided that extra sufficiency-type conditions on the remaining limit parameters are fulfilled. 
Theorem 3.4. The following properties hold.

(i) The limit equation (1.2) has two positive equilibrium points if $d<0$ and satisfies $|d|<$ $\left(\left(\gamma \mu(p / \alpha e)^{p}-1\right) K-(\mu-1)(p / \alpha) /((\mu-1) p+\alpha K)\right) p$, provided that $1>\gamma>(((\mu-1) p+$ $K \alpha) / \mu K \alpha)(\alpha e / p)^{p}, K>\max \left(0,(\mu-1) p / \mu \alpha\left[(p / \alpha e)^{p}-1 / \mu\right]\right)$.

(ii) If $d=0$, then the limit equation (1.2) has a zero equilibrium point and another positive equilibrium points, and $K>(\mu-1)(p / \alpha) /\left(\gamma \mu(p / \alpha e)^{p}-1\right)$.

Proof. (i) One gets from (3.6) for $d<0$ that

$$
h(x)=(\mu-1) x^{2}+(K+(\mu-1)|d|-\gamma \mu K \zeta(x)) x+K|d| .
$$

For $x=p / \alpha>0$ where $\zeta(x)$ reaches its maximum value $(p / \alpha e)^{p}$, and

$$
h\left(\frac{p}{\alpha}\right)=\left((\mu-1) \frac{p}{\alpha}+K\right) \frac{p}{\alpha}+\left((\mu-1) \frac{p}{\alpha}+K\right)|d|-\frac{\gamma \mu K}{e^{p}}\left(\frac{p}{\alpha}\right)^{p+1}<0
$$

if $1>\gamma>(((\mu-1) p+K \alpha) / \mu K \alpha)(\alpha e / p)^{p}$ subject to $(p / \alpha e)^{p}>((\mu-1) p+K \alpha) / \mu K \alpha=(\mu-$ 1) $p / \mu K \alpha+1 / \mu$ for $K>0 \Leftrightarrow K>\max \left(0,(\mu-1) p / \mu \alpha\left[(p / \alpha e)^{p}-1 / \mu\right]\right)$. Since $h(0)=K|d|>$ $0, h(\infty)=\lim _{x \rightarrow \infty} h(x)=\infty, h(p / \alpha)=((\mu-1)(p / \alpha)+K)(p / \alpha)+((\mu-1)(p / \alpha)+K)|d|-$ $\left(\gamma \mu K / e^{p}\right)(p / \alpha)^{p+1}<0$ for $x=p / \alpha \in \mathbf{R}_{0+}$ and $h: \mathbf{R}_{0+} \rightarrow \mathbf{R}$ is a real continuous function, there exist $\bar{x}_{1,2} \in \mathbf{R}_{+}$with $\bar{x}_{2}>\bar{x}_{1}$ such that $h\left(\bar{x}_{i}\right)=0, i=1,2$ which are equilibrium points of the limit equation (1.2) and the proof is complete.

(ii) The proof is similar to that of (i) by noting that the particular condition $|d|=0<$ $\left(\left(\left(\gamma \mu(p / \alpha e)^{p}-1\right) K-(\mu-1)(p / \alpha)\right) /((\mu-1) p+\alpha K)\right) p$ is guaranteed by the given constraint on $K$.

The following result proves that if there is only one equilibrium point $(d>0)$ of the limit equation, then it is a globally stable attractor. Also, if there are two equilibrium points ( $d \leq 0$ with sufficiently small $|d|$ plus extra conditions on the parameters), then the smaller equilibrium point is locally unstable while the largest one is a globally stable attractor.

Theorem 3.5. The following properties hold.

(i) If $d>0$, then the unique positive equilibrium point is a global stable attractor.

(ii) If $d \leq 0$ and of sufficiently small modulus, satisfying the constraints of Theorem 3.4, then there are two nonnegative distinct equilibrium points. The smallest one is locally unstable if $p=0$, and locally stable if $p>0$ while the largest one is a global stable attractor.

Proof. (i) Consider the initial value problem $x_{i}=\hat{x}_{i} \geq 0(i=0,1)$. Thus, one has for $d>0$

$$
0<d \leq x_{k+1}=\frac{\mu K e^{-\alpha x_{k}} x_{k-1}}{K+(\mu-1) x_{k-1}}+d \leq \widehat{x}_{k+1} \leq \frac{\mu K e^{-\alpha \widehat{x}_{k}}}{\mu-1}+d \leq \limsup _{k \rightarrow \infty} \widehat{x}_{k} \leq \frac{\mu K}{\mu-1}+d<\infty .
$$

Thus,

$$
0<d \leq \liminf _{k \rightarrow \infty} x_{k} \leq \limsup _{k \rightarrow \infty} x_{k} \leq \frac{\mu K}{\mu-1}+d<\infty .
$$


The equilibrium point is positive and unique from Theorem 3.1(i) and within $[d, \sqrt{d K /(\mu-1)}] \subset[d, \mu K /(\mu-1)+d]$ from Theorem 3.3(i). Assume that the equilibrium point is locally unstable. Then, the stability constraints (3.18) do not jointly hold. Since (3.26) holds and the equilibrium is locally unstable, a stable limit oscillatory solution has to exist subject to (3.26). Then, there is $\theta \in \mathbf{N}$ (the oscillation period) such that $0<d \leq \lim _{k \rightarrow \infty} x_{k+k_{1}}=\bar{x}_{k+k_{1}} \leq \mu K /(\mu-1)+d<\infty$, for all $k_{1} \in \bar{\theta}$. Now, build the real vector $x_{k}^{(0)}:=\left(x_{k}, x_{k-1}, \ldots, x_{k-\theta+1}\right)^{T} \rightarrow \bar{x}_{k}^{(0)}$ as $k \rightarrow \infty$. The linearization about the limit oscillation (limit cycle) is defined by the dynamics $z_{k+1}^{(0)}=A z_{k}^{(0)}$ where $A \in \mathbf{R}^{\theta \times \theta}$ is block-partitioned

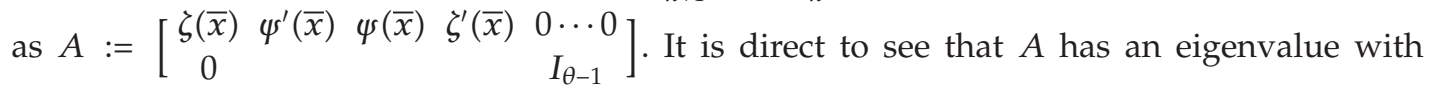
modulus greater than unity if and only if (3.16) has a zero with modulus greater than unity since they coincide. Therefore, the limit cycle is unstable which leads to a contradiction to the fact that it should exist and be stable. Then, the unique equilibrium point is locally stable and there is no stable limit cycle. Since the equilibrium point is stable and unique, it is also a global stable attractor and Property (i) has been proved.

(ii) If $d=0$, then $\bar{x}=0$ is an equilibrium point. For any small perturbation population at sampled time $k$, which has to be positive if $p=0, x_{j}>0$ for $j \in \bar{J} \backslash \bar{k}$ for some finite integer $J>k$ from inspection of the limit equation (1.2). Then, the zero equilibrium is locally unstable. An alternative proof is the test of the stability conditions (3.18) for $p=\bar{x}=0$. The second condition $\left|\psi(0) \zeta^{\prime}(0)\right|+\zeta(0) \psi^{\prime}(0)=\zeta(0) \psi^{\prime}(0)=\gamma \mu>1$ is violated so that $\bar{x}=0$ is locally unstable. For $\bar{x}=0$ and $p>0$, both stability conditions are fulfilled as $1+\zeta(0) \psi^{\prime}(0)=1>0$, $\left|\psi(0) \zeta^{\prime}(0)\right|+\zeta(0) \psi^{\prime}(0)=0<1$ so that the zero equilibrium point is locally stable. The largest equilibrium point is a positive global stable attractor from Property (i). If $d \leq 0$ and satisfies the conditions of Theorem 3.4, then there are two positive equilibrium points. The largest one is a stable global attractor proved under similar considerations as those used to prove Property (i).

\section{Existence of oscillatory solutions of the nonstationary-modified Beverton-Holt equation}

The main motivation of the study of this section is that very commonly the evolution equation of populations in biology problems follows oscillatory seasonal patterns according to reproduction needs, food supply from the environment, or metabolic cycles of the species [13]. In Theorem 2.8, it has been proved that

$$
\begin{aligned}
0<s_{k} & =\prod_{i=0}^{k}\left[\rho_{i}\right] s_{0}+\sum_{i=0}^{k-1} \prod_{j=i+1}^{k-1}\left[\rho_{j}\right] \eta_{i} \\
& =\prod_{i=0}^{k}\left[\rho_{i}\right] s_{0}+\sum_{i=0}^{k-1} \prod_{j=i+1}^{k-1}\left[\rho_{j}\right]\left(v_{i}-\omega_{i}\right) \leq \infty, \quad \forall k \in \mathbf{N},
\end{aligned}
$$

where $\rho_{i}:=1 / \gamma_{i} \mu_{i-1} \zeta_{i}, \omega_{i}:=K_{i-1} s_{i-1} \delta_{i} d_{i} / \rho_{i}^{-1}\left(\rho_{i}^{-1}+\delta_{i} d_{i}\right)$ and $\delta_{i}:=K_{i-1} s_{i-1}+\mu_{i-1}-1$, which implies and it is implied by $x_{k} \in[0, \infty)$; for all $k \in \mathbf{N}$. Furthermore, if $d_{k}>0$; for all $k \in \mathbf{N}$ then the above upper-bound is finite which implies and it is implied by $x_{k} \in[0, \infty)$; for all $k \in \mathbf{N}$. It is of interest to investigate when the solution of the modified Beverton-Holt equation 
is oscillatory equivalent to the solution of its inverse to be oscillatory. It has to be pointed out that the generation of limit oscillatory solutions being independent of the initial conditions is very important in some engineering problems, like, for instance, all those associated with the synthesis of electronic oscillators (see, e.g., [28]). To that end in the context of the modified Beverton-Holt equation (1.2), define the discrete functions $\chi_{x}: \mathbf{N}_{0}^{2} \rightarrow \mathbf{R}$ and $\overline{\chi_{x}}: \mathbf{N}_{0} \times \mathbf{N}^{2} \rightarrow \mathbf{R}$ as follows:

$$
\begin{gathered}
\chi_{x}(j, k):=x_{k}-x_{j}, \\
\bar{\chi}_{x}(k, k+j, k+j+\ell):=\chi_{x}(k, k+j) \chi_{x}(k, k+j+\ell)=\left(x_{k+j}-x_{k}\right)\left(x_{k+j+\ell}-x_{k}\right),
\end{gathered}
$$

respectively. Thus, precise definitions of what is meant by oscillatory solution of (1.2) are given.

Definition 4.1. A particular solution of (1.2) is strongly oscillatory if for any $k \in \mathbf{N}_{0}$, there exist two finite natural numbers $N_{i}(k) ; i=1,2$, depending on $k$, such that

$$
\bar{X}_{x}\left(k, k+N_{1}(k), k+N_{1}(k)+N_{2}(k)\right)<0 .
$$

Definition 4.2. A particular solution of (1.2) is weakly oscillatory if for any $k \in\left[\bar{k}_{1}, \bar{k}_{2}\right]=\bar{k}_{2} \backslash$ $\overline{k_{1}-1} \subset \mathbf{N}_{0}$ and some $\bar{k}_{1} \in \mathbf{N}_{0}$ and $k_{2}\left(\geq k_{1}\right) \in \mathbf{N}_{0}$, there exist two finite natural numbers $N_{i}(k) ; i=1,2$, depending on $k$, such that

$$
\bar{X}_{x}\left(k, k+N_{1}(k), k+N_{1}(k)+N_{2}(k)\right)<0 .
$$

It turns out that any oscillation of the Beverton-Holt equation implies, and is implied by, an oscillation of the solution of its inverse. Definitions 4.1 and 4.2 imply two alternate changes of sign at some three samples everywhere for the solution sequence (strong oscillation) or within some finite interval (weak oscillation), respectively. For each interval $\left[k, k+N_{1}(k), k+\right.$ $N_{1}(k)+N_{2}(k)$ ], only one oscillation test is needed. If $x_{k}=0$, then either the population is extinguished and remains at the zero equilibrium point or it is recovering positive values at the next samples generated by immigration so that the oscillation existence text can be performed later on formally.

Assertion 4.3. $\bar{X}_{x}\left(k, k+N_{1}(k), k+N_{1}(k)+N_{2}(k)\right)<0$ for $k \in \mathbf{N}_{0}, N_{i}(k) \in \mathbf{N}(i=1,2) \Leftrightarrow$ $\bar{X}_{s}\left(k, k+N_{1}(k), k+N_{1}(k)+N_{2}(k)\right)<0$ for $k \in \mathbf{N}_{0}, N_{i}(k) \in \mathbf{N}(i=1,2)$.

Proof. It is direct from $s_{k}=x_{k}^{-1}$, for all $k \in \mathbf{N}_{0}$ if $x_{k}>0$. If $x_{k}=0$, either the population is extinguished for all samples and no test for oscillation applies or some population is recovered from immigration (positive disturbance) at future samples and the inverse Beverton-Holt equation can be defined.

Assertion 4.4. The subsequent technical result holds:

$$
\bar{X}_{x}\left(k, k+N_{1}(k), k_{1}\right)<0 \wedge \bar{X}_{x}\left(k_{1}(k), k_{1}(k)+N_{1}\left(k_{1}(k)\right), k_{1}(k)+N_{1}\left(k_{1}(k)\right)+N_{2}\left(k_{1}(k)\right)\right)<0
$$

with $k_{1}(k):=k+N_{1}(k)+N_{2}(k)$ for some $k \in \mathbf{N}_{0}, N_{i}(k) \in \mathbf{N}, N_{i}\left(k_{1}\right) \in \mathbf{N}(i=1,2)$

$$
\Longleftrightarrow \exists N_{i}(k+j) \in \mathbf{N}(i=1,2): \bar{X}_{x}\left(k+j, k+N_{1}(k+j)+N_{2}(k+j)\right)<0,
$$

for all $j\left(\leq N_{1}(k)+N_{2}(k)\right) \in \mathbf{N}$ such that $x_{k+j}>0, N_{i}(k) \in \mathbf{N}$. 
Proof. Note that

$$
\begin{aligned}
\operatorname{sign}\left(x_{k+N_{1}(k)}-x_{k}\right) & \neq \operatorname{sign}\left(x_{k+k_{1}(k)}-x_{k}\right), \\
\operatorname{sign}\left(x_{k+N_{1}(k)}-x_{k}\right) & \neq \operatorname{sign}\left(x_{k+k_{1}(k)}-x_{k+N_{1}(k)}\right), \\
\operatorname{sign}\left(x_{k_{1}(k)+N_{1}\left(k_{1}(k)\right)}-x_{k_{1}(k)}\right) & \neq \operatorname{sign}\left(x_{k_{1}(k)+N_{1}\left(k_{1}(k)\right)+N_{2}\left(k_{1}(k)\right)}-x_{\left.k_{1}(k)+N_{1}\left(k_{1}(k)\right)\right),},\right. \\
\operatorname{sign}\left(x_{k_{1}(k)+N_{1}\left(k_{1}(k)\right)}-x_{k_{1}(k)}\right) & \neq \operatorname{sign}\left(x_{k_{1}(k)+N_{1}\left(k_{1}(k)\right)+N_{2}\left(k_{1}(k)\right)}-x_{k_{1}(k)+N_{1}\left(k_{1}(k)\right)}\right) .
\end{aligned}
$$

Thus, it is obvious that there exist $N_{i}(k+j) \in \mathbf{N}(i=1,2): \bar{X}_{x}\left(k+j, k+N_{1}(k+j)+N_{2}(k+j)\right)<0$, for all $j \in \mathbf{N}$ such that $x_{k+j}>0$, for all $j\left(\leq N_{1}(k)+N_{2}(k)\right) \in \mathbf{N}$, since it always exist by construction at least one $N_{1}(k+j) \leq N_{1}\left(k_{1}(k)\right)$ and at least one $N_{2}(k+j) \leq N_{2}\left(k_{1}(k)\right)$, for all $j \in \mathbf{N}$ such that $x_{k+j}>0$. If $x_{k+j}=0$, for all $j \neq k, k+N_{1}(k), k+N_{1}(k)+N_{2}(k)$, then there are always at least three sign changes in the interval $\left[k, k+N_{1}(k)+N_{2}(k)\right]$ and the proof also follows.

Assertions 4.3-4.4 imply directly the following result which is concerned with the simplification of the test for strong oscillatory solutions.

Assertion 4.5. Assume that there exists a subset of infinite cardinal of the set of natural numbers $S_{j}:=\left\{k_{j} \in \mathbf{N}_{0}: 1 \leq k_{j+1}-k_{j}<\infty\right\} \subset \mathbf{N}_{0}$ such that

$$
\begin{gathered}
\bar{X}_{x}\left(k, k+N_{1}(k), k+N_{1}(k)+N_{2}(k)\right)<0 \quad \forall k \in S_{j} \text { for some } N_{i}(k) \in \mathbf{N}(i=1,2) \\
\Longleftrightarrow \bar{X}_{s}\left(k, k+N_{1}(k), k+N_{1}(k)+N_{2}(k)\right)<0 \quad \text { for } k \in \mathbf{N}_{0}, N_{i}(k) \in \mathbf{N}(i=1,2)
\end{gathered}
$$

and some solution $\left\{x_{k}\right\}_{1}^{\infty}$ generated by initial conditions $x_{-1} \geq 0, x_{0}>0$. Then, such a solution is strongly oscillatory. The converse is also true.

The main result of this section follows.

Theorem 4.6. A solution of the modified Beverton-Holt equation (1.2), subject to initial conditions $x_{-1} \geq 0, x_{0}>0$, is strongly oscillatory if and only if there exists an infinite sequence of triples $\left\{k_{i}, N_{1}\left(k_{i}\right), N_{2}\left(k_{i}\right)\right\}_{i \in \mathbf{N}_{0}} \in \mathbf{N}^{3}$, where $k_{i+1}=k_{i}+N_{1}\left(k_{i}\right)+N_{2}\left(k_{i}\right) \in S \subset \mathbf{N}$ for some finite $k_{0} \in \mathbf{N}_{0}$, for all $i \in \mathbf{N}_{0}$ with $\mathbf{N} \ni N_{j}\left(k_{i}\right)<\infty(j=1,2)$ such that

$$
S_{k_{i}} S_{k_{i}+N_{1}\left(k_{i}\right)} S_{k_{i}+N_{1}\left(k_{i}\right)+N_{2}\left(k_{i}\right)}>0
$$

and, furthermore,

$$
\begin{aligned}
s_{k_{i}+N_{1}\left(k_{i}\right)+N_{2}\left(k_{i}\right)}<\frac{s_{k_{i}}\left(s_{k_{i}+N_{1}\left(k_{i}\right)}-s_{k_{i}}\right)}{s_{k_{i}+N_{1}\left(k_{i}\right)}-s_{k_{i}}}=\frac{s_{k_{i}+N_{1}\left(k_{i}\right)}-s_{k_{i}}}{\kappa_{i}-1} \\
\quad \Longleftrightarrow x_{k_{i}+N_{1}\left(k_{i}\right)+N_{2}\left(k_{i}\right)}>\frac{x_{k_{i}}-x_{k_{i}+N_{1}\left(k_{i}\right)}}{x_{k_{i}}\left(x_{k_{i}}-x_{k_{i}+N_{1}\left(k_{i}\right)}\right)} \\
\Longleftrightarrow\left(s_{k_{i}+N_{1}\left(k_{i}\right)+N_{2}\left(k_{i}\right)}<s_{k_{i}}<s_{k_{i}+N_{1}\left(k_{i}\right)}\right) \vee\left(s_{k_{i}+N_{1}\left(k_{i}\right)+N_{2}\left(k_{i}\right)}>s_{k_{i}}>s_{k_{i}+N_{1}\left(k_{i}\right)}\right) \\
\Longleftrightarrow\left(x_{k_{i}+N_{1}\left(k_{i}\right)+N_{2}\left(k_{i}\right)}>x_{k_{i}}>x_{k_{i}+N_{1}\left(k_{i}\right)}\right) \vee\left(x_{k_{i}+N_{1}\left(k_{i}\right)+N_{2}\left(k_{i}\right)}<x_{k_{i}}<x_{k_{i}+N_{1}\left(k_{i}\right)}\right),
\end{aligned}
$$

where $\kappa_{i}:=s_{k_{i}+N_{1}\left(k_{i}\right)} / s_{k_{i}}$. 
Proof. Expand $\bar{X}_{S}\left(k_{i}, k_{i}+N_{1}\left(k_{i}\right), k_{i}+N_{1}\left(k_{i}\right)+N_{2}\left(k_{i}\right)\right)$ using Definition 4.1 to obtain

$$
\begin{aligned}
& \bar{X}_{S}\left(k_{i}, k_{i}+N_{1}\left(k_{i}\right), k_{i}+N_{1}\left(k_{i}\right)+N_{2}\left(k_{i}\right)\right) \\
& \quad=s_{k_{i}}\left(s_{k_{i}}-s_{k_{i}+N_{1}\left(k_{i}\right)}-s_{k_{i}+N_{1}\left(k_{i}\right)+N_{2}\left(k_{i}\right)}\right)+s_{k_{i}+N_{1}\left(k_{i}\right)} s_{k_{i}+N_{1}\left(k_{i}\right)+N_{2}\left(k_{i}\right)}<0 .
\end{aligned}
$$

Then, the proof follows directly from Assertions 4.4-(4.5) and direct computations since (4.11) is equivalent to the four equivalent propositions for the existence of strong oscillatory solution.

Remark 4.7. Note that the statement after the disjunction symbol in the third proposition in Theorem 4.6 for existence of strong oscillation may be expanded as follows:

$$
\begin{aligned}
& \frac{1}{\gamma_{k_{i}+N_{1}\left(k_{i}\right)-1} \mu_{k_{i}+N_{1}\left(k_{i}\right)-2} \zeta_{k_{i}+N_{1}\left(k_{i}\right)-2}}\left(\prod_{k=k_{i}}^{k_{i}+N_{1}\left(k_{i}\right)-2}\left[\rho_{j}\right]\right) \\
& \quad+\sum_{j=0}^{k_{i}+N_{1}\left(k_{i}\right)-1} \prod_{\ell=j+1}^{k_{i}+N_{1}\left(k_{i}\right)-1}\left[\rho_{\ell}\right]\left(\frac{\eta_{j}}{s_{k_{i}}}\right)>1>s_{k_{i}+N_{1}\left(k_{i}\right)+N_{2}\left(k_{i}\right)} \\
& =\frac{1}{\gamma_{k_{i}+N_{1}\left(k_{i}\right)+N_{2}\left(k_{i}\right)-1} \mu_{k_{i}+N_{1}\left(k_{i}\right)+N_{2}\left(k_{i}\right)-2} \zeta_{k_{i}+N_{1}\left(k_{i}\right)+N_{2}\left(k_{i}\right)-2}} \\
& =\left(\prod_{k=k_{i}}^{k_{i}+N_{1}\left(k_{i}\right)+N_{2}\left(k_{i}\right)-2}\left[\rho_{j}\right]\right)+\sum_{j=0}^{k_{i}+N_{1}\left(k_{i}\right)+N_{2}\left(k_{i}\right)-1} \sum_{k_{i}+N_{1}\left(k_{i}\right)+N_{2}\left(k_{i}\right)-1}\left[\rho_{\ell}\right]\left(\frac{\eta_{j}}{s_{k_{i}}}\right)
\end{aligned}
$$

since $\zeta(x)$ varies from zero to infinity, it is always possible to have strong oscillatory solutions by accomplishing with the inequalities with appropriate parameterizations of combinations of the harvesting quota and intrinsic growth rate at certain samples sufficiently far away from each former test for oscillation (see also Assertion 4.4). Similar considerations apply for the reverse inequalities guaranteeing also strong oscillatory solutions conditions for weak oscillatory solutions are similar but they only apply on some finite interval.

\section{Numerical example}

In this section, the evolution of a population of aphids is investigated under the modified generalized Beverton-Holt equation through a numerical tested example. A brief empirical description of those species follows. Aphids, or plant lice, are small, soft-bodied, pear-shaped insects which are commonly found on nearly all indoor and outdoor plants, as well as vegetables, field crops, and fruit trees. Most of them are about 1/10 inch long. They feed on plants by piercing them with syringe-like mouths pars and sucking the sap. Their diet is rich in carbohydrates and deficient in amino acids. Some of these amino acids cannot be synthesized by the insect but are supplied by the intracellular symbiont bacteria Buchnera aphidicola. Such a symbiont lives inside huge host cells (bacteriocytes) of which there are about 60-80 per individual and are transmitted to eggs through generations. Aphids have unusual and complex life cycles which allow them to build up huge levels of population in very short periods of time. Furthermore, they overwinter as fertilized eggs. Nymphs which hatch from these eggs become wingless females (stem mothers) which reproduce without mating holding 


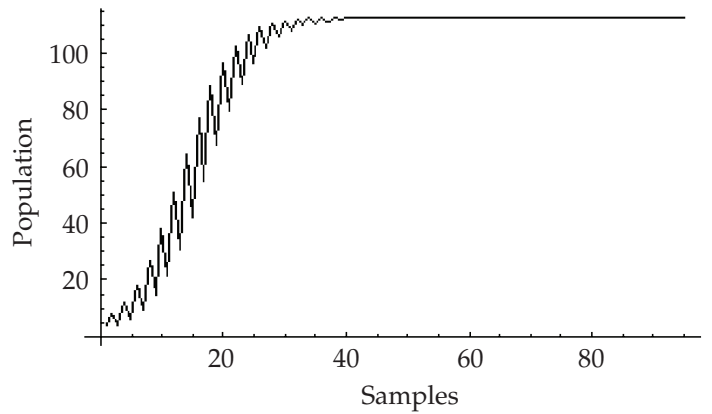

Figure 1: $\mu=2, K=200, d=0, p=0.01, \alpha=0.002$, and $\gamma=0.8$.

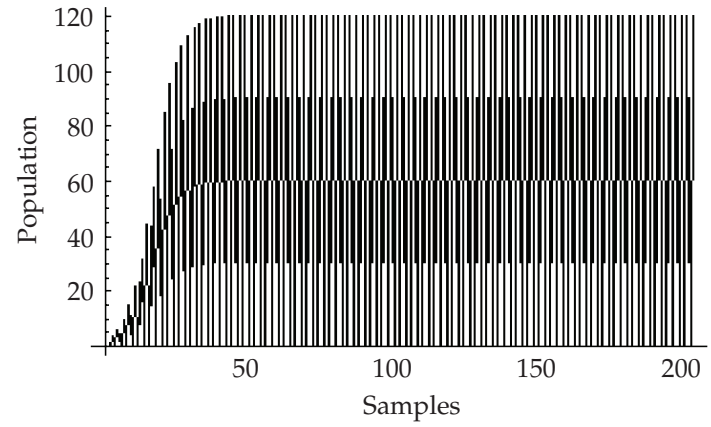

Figure 2: $\mu=2, K=200, d=0, p=0.01, \alpha=0.001$, and $\gamma=0.8$.

new amounts of eggs in their bodies until hatching born alive youths. This pattern continues for as long as conditions are favorable. When the days get shorter in the fall and there are cooler temperatures, a generation appears which includes both males and females which are the production of fertilized eggs which overwinter [14]. As a short summary of the above description, it can be emphasized that the populations grow very fast in short periods of time during the same year and they also decrease fast to reach low survival thresholds but not up to extinction.

A common mathematical model for the population evolution of aphids is given in [13] as follows:

$$
a_{k+1}=f r(1-m) a_{k}=[f r(1-m)]^{k+1} a_{0},
$$

where $a_{k}$ is the number of adult female aphids in the $k$ th generation, $p_{k}=f a_{k}$ is the number of progeny in the $k$ th generation, $m$ is the fractional mortality of the young aphids, $f$ is the number of progeny per female aphid and $r$ is the ratio of female aphids to total adult aphids. The above parameters are all nonnegative by nature. The model might be generalized to extend the above parameters to be varying sequence. From standard stability results the model is globally asymptotically stable (and also exponentially stable) if and only if $g:=f r(1-m)<1$, globally stable with the population being constant if $g=1$ and unstable (with the solution diverging at exponential rate) if $g>1$. It turns out that such a description does not fix properly the above empirical description from biology knowledge. Exhaustive simulation 


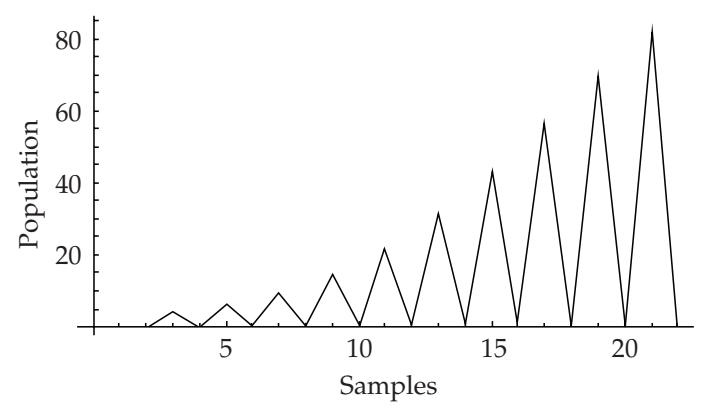

Figure 3: $\mu=2, K=200, d=0, p=0.01, \alpha=0.001$, and $\gamma=0.8$

via the modified generalized Beverton-Holt equation shows that the nontrivial solutions are always bounded and positive as expected. They have equilibrium points which depend on parameters. For instance, for zero harvesting quota, zero disturbance contribution, and very small or zero powers $p$ of the penalty term, the solutions reach an equilibrium which lies below that reached by the standard Beverton-Holt model, defined by the carrying capacity. However, there are several cycles of alternate fast increase/decrease of the populations during the transients. This model property is useful to describe the above biological behavior during one year of population evolution. If the parameters are readjusted, the solution changes accordingly while keeping a similar shape. For instance, the upper limit is smaller as the penalty term increases which also produces more abrupt cycles of increase/decrease of the population.

Figure 1 describes the population evolution for constant parameterizations $\mu=2, K=$ 200, $d=0, p=0.01, \alpha=0.002$, and $\gamma=0.8$. It is seen that the upper limit is smaller than the level of 200 corresponding to a standard Beverton-Hold equation, governed by $\mu$ and $K$, and also smaller than the level of 160 , corresponding to a (unmodified) generalized Beverton-Holt equation, governed by $\mu, K, \gamma$, and $d=0$. The reason is that a penalty term is introduced for the overpopulation of aphids. The penalty term translates into a certain level of population control since the population individuals compete in their habitat. The transient shows alternate cycles of increase/decrease of population levels. If $\alpha=0.001$, with the remaining parameters being identical, then the upper limit increases as expected since both the standard and unmodified generalized Beverton-Holt equations are parameterized by a carrying capacity of 200. The population evolution is displayed in Figure 2 where the existence of the upper limit is obvious and a zoom for the first part of transient exhibiting the alternate cycles is given in Figure 3. Finally, Figure 4 shows the sample-to-sample ratio between the population evolution of the modified generalized Beverton-Holt equation to the standard one over a transient of 30 samples for zero harvesting quota.

On the other hand, Figures 5 and 6 display the population evolution under moderate disturbance contribution consisting of joint independent consumption plus net migration fixed to $1 / 10$ of the initial population with harvesting quotas of $20 \%$ and $60 \%$, respectively. Those quotas can be interpreted in the plagues context as the use of pesticides to fight the plague. In the first case, the population grows slightly for each cycle, but exhaustive simulation for more samples demonstrates that the population remains bounded to smaller values than the carrying capacity, as expected. In the second case, it is shown that the levels of population decrease at each successive life cycle. 


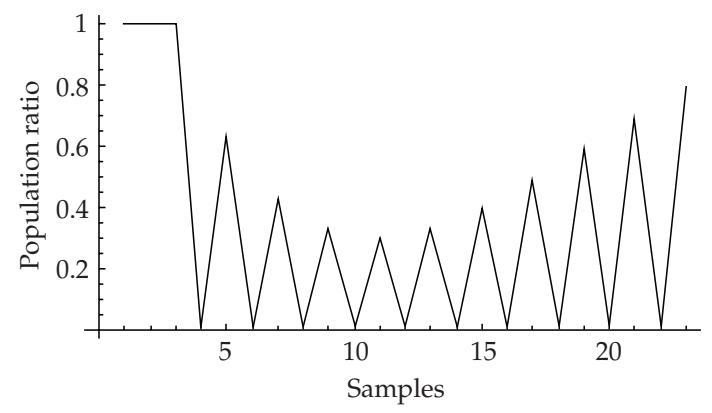

Figure 4: Relative comparison with the standard Beverton-Holt equation.

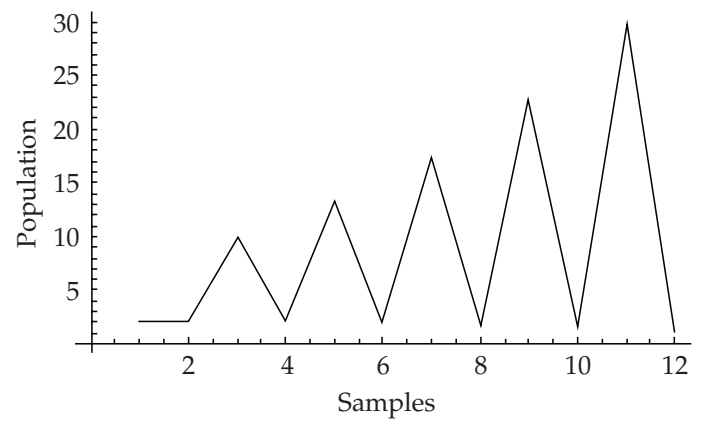

Figure 5: $\mu=2, K=200, \gamma=0.8, d=0.1 x(0), \alpha=0.1, p=10$.

\section{Conclusions}

This paper has been devoted to the study of stability and equilibrium point of a proposed modified generalized Beverton-Holt equation in ecology. Such an equation is governed, in general, by six parametrical sequences, namely,

(i) the intrinsic growth rate and the environment carrying capacity which define the standard Beverton-Holt equation,

(ii) the harvesting quota and the disturbance contribution which, in addition, to the two former ones parameterize the generalized Beverton-Holt equation,

(iii) a penalty term for the eventual overpopulation which includes, in general, the product of a potential term and a decreasing exponential one of the previous population.

It has been proved that the proposed model is Lyapunov stable and possesses stable equilibrium points. It has also been proved that even under constant parameterizations the model can exhibit an oscillatory behavior to describe the alternative cycles of increasing/decreasing levels of population evolution. In this sense, it can be useful to correct the foreseen evolution through the standard Beverton-Holt equation which, under nonextinction conditions obtained from a carrying capacity greater than unity, leads to the population convergence to a finite set point limit defined by the environment carrying capacity. It can also be useful to introduce a correction of the behavior foreseen by its limiting equation, which is the Ricker model, 


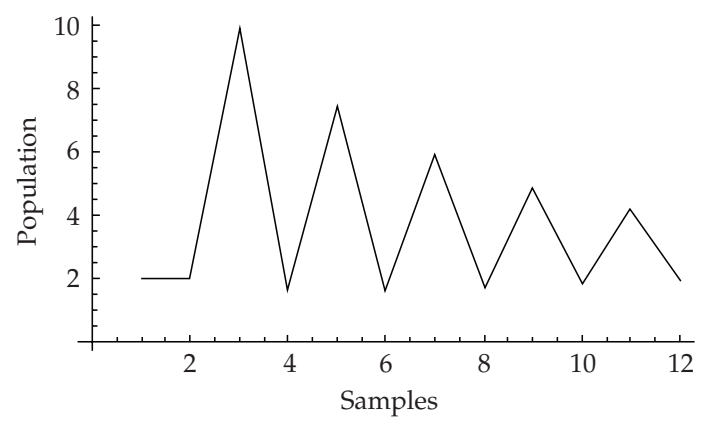

Figure 6: $\mu=2, K=200, \gamma=0.4, d=0.1 x(0), \alpha=0.1, p=10$.

which leads also to a limit set point being typically smaller than that associated with the standard Beveerton- Holt equation. The modified generalized Beverton-Holt equation is also shown to be useful to describe during its transient alternate cycles of the population levels for certain populations of insects since the solution is bounded and extinction-free under standard parameterization conditions so that it exhibits an oscillatory behavior within finite positive lower and upper bounds. In this context and in order to show its usefulness the proposed model has been applied to study a population of aphids which exhibit alternate cycles of increase and decrease of population and which state in latent levels of populations against very adverse conditions of their habitat.

\section{Acknowledgments}

The author is very grateful to MCYT by its partial support of this work through Grant no. DPI2006-00714 and to the Basque Government by its support of this work via Research Grants: Research Groups nos. IT-269-07, SAIOTEK SPE07UN04, and SAIOTEK SPED06UN10. $\mathrm{He}$ is also very grateful to the reviewers by their interesting comments which have helped to improve the content of the original manuscript.

\section{References}

[1] S. Elaydi and R. J. Sacker, "Global stability of periodic orbits of non-autonomous difference equations and population biology," Journal of Differential Equations, vol. 208, no. 1, pp. 258-273, 2005.

[2] V. L. Kocic, "A note on the nonautonomous Beverton-Holt model," Journal of Difference Equations and Applications, vol. 11, no. 4-5, pp. 415-422, 2005.

[3] J. M. Cushing and S. M. Henson, "A periodically forced Beverton-Holt equation," Journal of Difference Equations and Applications, vol. 8, no. 12, pp. 1119-1120, 2002.

[4] J. M. Cushing and S. M. Henson, "Global dynamics of some periodically forced, monotone difference equations," Journal of Difference Equations and Applications, vol. 7, no. 6, pp. 859-872, 2001.

[5] S. Elaydi and R. J. Sacker, "Nonautonomous Beverton-Holt equations and the Cushing-Henson conjectures," Journal of Difference Equations and Applications, vol. 11, no. 4-5, pp. 337-346, 2005.

[6] S. Elaydi and R. J. Sacker, "Periodic difference equations, population biology and the Cushing-Henson conjectures," Mathematical Biosciences, vol. 201, no. 1-2, pp. 195-207, 2006.

[7] F. Chen and C. Shi, "Global attractivity in an almost periodic multi-species nonlinear ecological model," Applied Mathematics and Computation, vol. 180, no. 1, pp. 376-392, 2006.

[8] X. Meng, W. Xu, and L. Chen, "Profitless delays for a nonautonomous Lotka-Volterra predator-prey almost periodic system with dispersion," Applied Mathematics and Computation, vol. 188, no. 1, pp. 365-378, 2007. 
[9] L. White, F. White, Y. Luo, and T. Xu, "Estimation of parameters in carbon sequestration models from net ecosystem exchange data," Applied Mathematics and Computation, vol. 181, no. 2, pp. 864-879, 2006.

[10] R. R. Sarkar, B. Mukhopadhyay, R. Bhattacharyya, and S. Banerjee, "Time lags can control algal bloom in two harmful phytoplankton-zooplankton system," Applied Mathematics and Computation, vol. 186, no. 1, pp. 445-459, 2007.

[11] S. Stević, "A short proof of the Cushing-Henson conjecture," Discrete Dynamics in Nature and Society, Article ID 37264, 5 pages, 2006.

[12] L. Berezansky and E. Braverman, "On impulsive Beverton-Holt difference equations and their applications," Journal of Difference Equations and Applications, vol. 10, no. 9, pp. 851-868, 2004.

[13] L. Edelstein-Keshet, Mathematical Models in Biology, vol. 46 of Classics in Applied Mathematics, SIAM, Philadelphia, Pa, USA, 2005.

[14] S. Shigenobu, H. Watanabe, M. Hattori, Y. Sakaki, and H. Ishikawa, "Genome sequence of the endocellular bacterial symbiont of aphids Buchnera sp. APS," Nature, vol. 407, no. 6, pp. 81-86, 2000.

[15] E. M. Elabbasy, H. El-Metwally, and E. M. Elsayed, "On the difference equation $x_{n+1}=\left(a x_{n}-\right.$ $\left.b x_{n}\right) /\left(c x_{n}-d x_{n-1}\right)$, , Advances in Difference Equations, Article ID 82579, 10 pages, 2006.

[16] M. de la Sen and S. Alonso-Quesada, "Model matching via multirate sampling with fast sampled input guaranteeing the stability of the plant zeros: extensions to adaptive control," IET Control Theory $\mathcal{E}$ Applications, vol. 1, no. 1, pp. 210-225, 2007.

[17] S. Ozen, I. Ozturk, and F. Bozkurt, "On the recursive sequence $y_{n+1}=\left(\alpha+y_{n-1}\right) /\left(\beta+y_{n}\right)+\left(y_{n-1}\right) /\left(y_{n}\right)$," Applied Mathematics and Computation, vol. 188, no. 1, pp. 180-188, 2007.

[18] I. Ozturk, F. Bozkurt, and S. Ozen, "On the difference equation $y_{n+1}=\left(\alpha+\beta e^{-\gamma n}\right) /\left(\gamma+y_{n-1}\right)$," Applied Mathematics and Computation, vol. 181, no. 2, pp. 1387-1393, 2006.

[19] H. El-Metwally, E. A. Grove, G. Ladas, R. Levins, and M. Radin, “On the difference equation $x_{n+1}=$ $\alpha+\beta x_{n-1} e^{-x_{n}}, "$ Nonlinear Analysis: Theory, Methods \& Applications, vol. 47, no. 7, pp. 4623-4634, 2001

[20] E. Camouzis, E. Chatterjee, and G. Ladas, "On the dynamics of $x_{n+1}=\left(\delta x_{n-2}+x_{n-3}\right) /\left(A+x_{n-3}\right)$," Journal of Mathematical Analysis and Applications, vol. 331, no. 1, pp. 230-239, 2007.

[21] S. Stević, "On the recursive sequence $x_{n+1}=A+\left(x_{n}^{p}\right) /\left(x_{n-1}^{p}\right)$, " Discrete Dynamics in Nature and Society, Article ID 34517, 9 pages, 2007.

[22] E. Camouzis and G. Ladas, "Periodically forced Pielou's equation," Journal of Mathematical Analysis and Applications, vol. 333, no. 1, pp. 117-127, 2007.

[23] A. M. Amleh, E. A. Grove, G. Ladas, and D. A. Georgiou, "On the recursive sequence $x_{n+1}=\alpha+$ $\left(x_{n-1}\right) /\left(x_{n}\right), "$ Journal of Mathematical Analysis and Applications, vol. 233, no. 2, pp. 790-798, 1999.

[24] M. de la Sen, "Robust stabilization of a class of polytopic linear time-varying continuous systems under point delays and saturating controls," Applied Mathematics and Computation, vol. 181, no. 1, pp. 73-83, 2006.

[25] M. de la Sen, "Parameter dependent Lyapunov functions for robust stability of time-varying linear systems under point delays," Applied Mathematics and Computation, vol. 179, no. 2, pp. 612-621, 2006.

[26] M. de la Sen, "Robust stable pole-placement adaptive control of linear systems with multiestimation," Applied Mathematics and Computation, vol. 172, no. 2, pp. 1145-1174, 2006.

[27] M. de la Sen, "The environment carrying capacity is not independent of the intrinsic growth rate for subcritical spawning stock biomass in the Beverton-Holt equation," Ecological Modelling, vol. 204, no. 1-2, pp. 271-273, 2007.

[28] N. Luo and M. Delasen, "State feedback sliding mode control of a class of uncertain time delay systems," IEE Proceedings D: Control Theory and Applications, vol. 140, no. 4, pp. 261-274, 1993. 


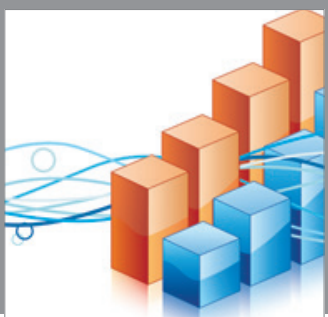

Advances in

Operations Research

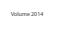

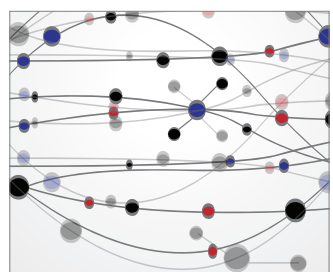

\section{The Scientific} World Journal
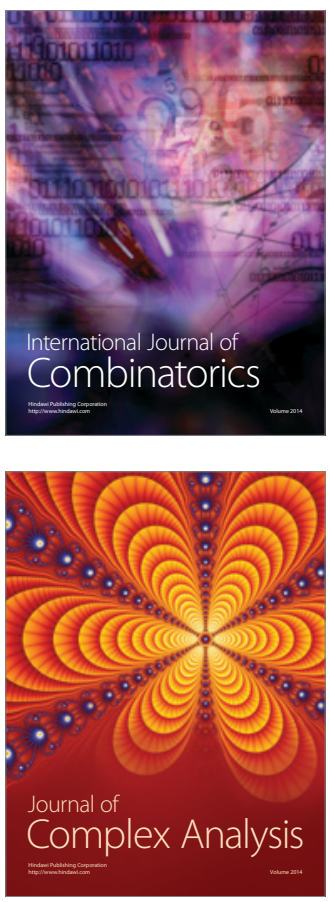

International Journal of

Mathematics and

Mathematical

Sciences
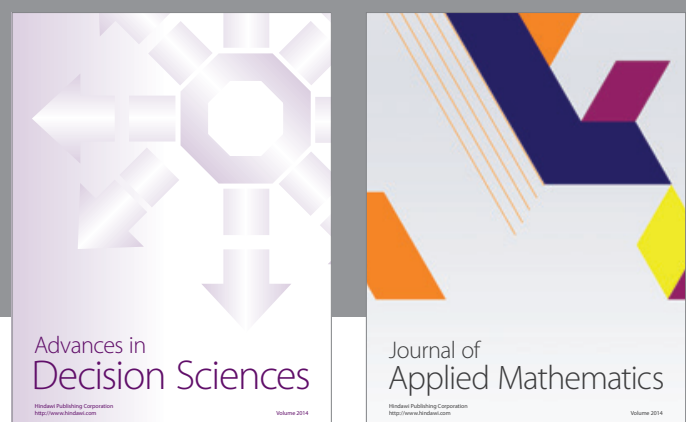

Journal of

Applied Mathematics
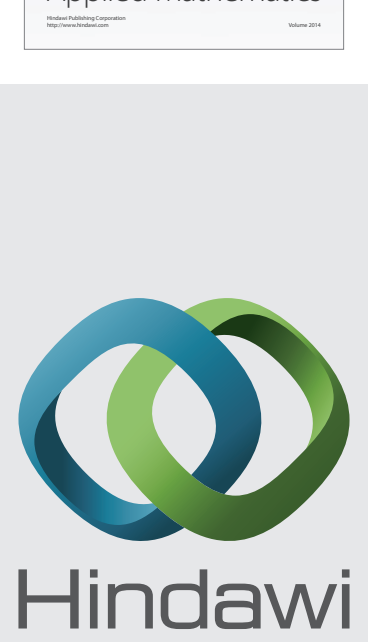

Submit your manuscripts at http://www.hindawi.com
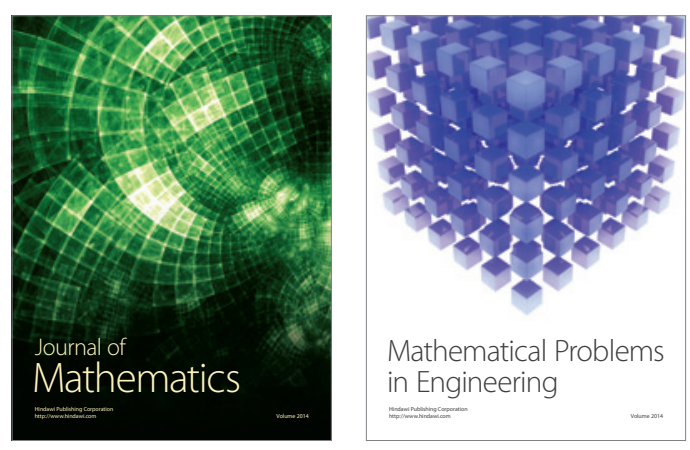

Mathematical Problems in Engineering
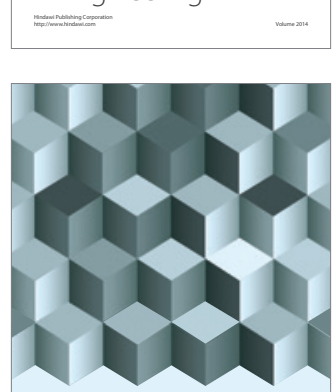

Journal of

Function Spaces
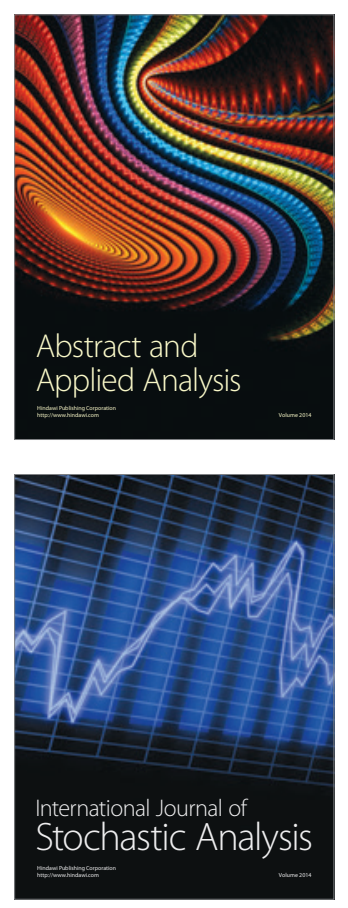

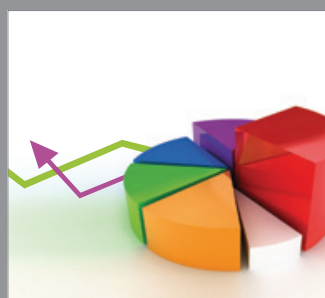

ournal of

Probability and Statistics

Promensencen
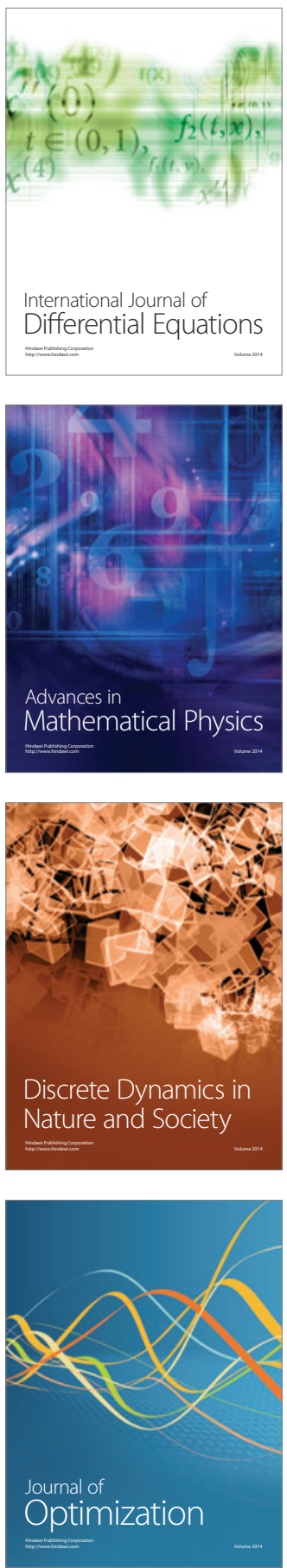